\title{
DNA Hypermethylation Modification Promotes the Development of Hepatocellular Carcinoma by Depressing the Tumor Suppressor Gene ZNF334
}

\section{Da-peng Sun}

Eastern Hepatobiliary Surgery Hospital

\section{Xiao-jie Gan}

Eastern Hepatobiliary Surgery Hospital

Lei Liu

Eastern Hepatobiliary Surgery Hospital

Yuan Yang

Eastern Hepatobiliary Surgery Hospital

Dong-yang Ding

Eastern Hepatobiliary Surgery Hospital

Wen Li

Eastern Hepatobiliary Surgery Hospital

Junyao Jiang

Guangzhou Institutes of Biomedicine and Health Chinese Academy of Sciences: Chinese Academy of

Sciences Guangzhou Institutes of Biomedicine and Health

\section{Wen-Bin Ding}

Eastern Hepatobiliary Surgery Hospital

\section{Ling-hao Zhao}

Eastern Hepatobiliary Surgery Hospital

\section{Guo-jun Hou}

Eastern Hepatobiliary Surgery Hospital

Jian Yu

Eastern Hepatobiliary Surgery Hospital

Jie Wang

Guangzhou Institutes of Biomedicine and Health Chinese Academy of Sciences: Chinese Academy of Sciences Guangzhou Institutes of Biomedicine and Health

Wei-ping Zhou ( $\nabla$ ehphwp@126.com )

Eastern Hepatobiliary Surgery Hospital

\section{Fu Yang}

second military medical university

\section{Sheng-xian Yuan}




\section{Research Article}

Keywords: DNA hypermethylation modifications, ZNF334, P53, hepatocellular carcinoma, inflammatory cancer transformation, tumor suppressor gene

Posted Date: October 27th, 2021

DOI: https://doi.org/10.21203/rs.3.rs-1000528/v1

License: (c) (1) This work is licensed under a Creative Commons Attribution 4.0 International License. Read Full License 


\section{Abstract}

Background: DNA methylation plays a pivotal role in the development and progression of tumors, but studies focused on the dynamic changes of DNA methylation in the development of hepatocellular carcinoma (HCC) are rare. This manuscript is aimed to construct pre- and early DNA methylation maps of liver cancer of the same genetic background, as well as to reveal the mechanism of epigenetics regulating gene expression during the development of liver cancer, thus providing new targets and clinical evidence for early diagnosis and shedding lights on the precise treatment for liver cancer.

Methods: The study includes 5 patients who were chronic hepatitis B virus infected, clinically diagnosed as primary liver cancer and pathologically diagnosed as early liver cancer with liver dysplastic nodules. Liver fibrosis tissues, dysplastic nodules and early HCC tissues from these patients have been used to measure DNA methylation.

Results: We report significant differences in the DNA methylation spectrum of three types of tissues. In the early stage of HCC, DNA hypermethylation of tumor suppressor genes is predominant. Additionally, DNA hypermethylation in the early stage of $\mathrm{HCC}$ changes the binding of transcription factor P53 to the promoter of tumor suppressor gene ZNF334, and inhibits the expression of ZNF334 at the transcription level. Furthermore, through a series of in vivo and in vitro experiments, we have clarified the exacerbation effect of tumor suppressor gene ZNF334 deletion in the occurrence of HCC. Combined with clinical data, we found that the overall survival and disease-free survival of patients with high ZNF334 expression are longer than the lower one.

Conclusions: We constructed a sequential map of DNA methylation modification during the occurrence of $\mathrm{HCC}$, and clarified the biological function and regulatory mechanism of the tumor suppressor gene ZNF334, which is regulated by related DNA methylation sites, and also provide new targets and clinical evidence for the early diagnosis and precise treatment of liver cancer.

\section{Background}

Primary liver cancer (PLC) is one of the most common malignant tumors with rapid development, low surgical resection rate, easy recurrence and metastasis, and high fatality rate[1-3]. Chronic inflammation of the liver caused by alcohol, hepatitis virus, metabolic disorders, etc. is generally considered to be the initiating factor for the occurrence of hepatocellular carcinoma (HCC)[4-7]. The inflammatory-cancer transformation of the liver requires a long fibrosis process, often from the liver dysplastic nodule (Dn) through a sequential process that gradually changes from benign to malignant[8]. It can be seen clinically that in some well-differentiated PLC, the tumor tissue is surrounded by high-grade Dn to form a "nod in the nodule" structure[9]. Therefore, the liver Dn is generally recognized as precancerous lesions that are closer to liver cancer than cirrhotic tissue[10]; however, few previous studies focused on the liver Dn, which is an important period of liver cancer in the early stage. In view of the strong metastatic characteristics of early liver cancer, research on precancerous lesions of liver cancer can better help find 
the driving events or molecules of the HCC, which is a significant premise to realize the early diagnosis and better treatment of liver cancer.

Epigenetic modifications, including histone modification, DNA methylation modification, chromatin remodeling, and non-coding RNA regulation, are crucial regulatory mechanisms for cells to adapt to changes in the external environment[11, 12]. Studies have shown that DNA methylation modification disorder is a common feature in PLC $[13,14]$. Genome-wide hypomethylation in HCC can cause genome instability, which in turn leads to mutations or expression alterations of tumor-related genes[15]. In the meanwhile, local abnormal methylation of tumor-related genes can inhibit the expression of tumor suppressor genes (TSGs) or promote the expression of oncogenes, thereby promoting the occurrence and development of liver cancer[16]. Previous studies have also shown that during the occurrence of hepatitis $B$ virus-related liver cancer, the abnormality of DNA methylation modification as well as malfunction of key enzymes can lead to the inactivation of TSG and the activation of oncogenes, accelerating the development of liver cancer[17, 18]. DNA methylation modification may have different patterns in different stages of liver cancer. In the comparison of early liver cancer and advanced liver cancer, the lineage of DNA methylation is the most extensively different, which also indicates the abnormalities of the methylation modification in HCC are secondary changes after the occurrence of liver cancer[19].

However, previous studies usually prefer the same type of tissue from different patients for research. Although the tissue types are the same, the genetic background is not completely in common. In the sequential process from hepatitis to cirrhosis to cancer, the continuous changes in the genome are ignored and we can only analyze the changes in the process of "inflammation-to-cancer transformation" truncated from different time points, which lacks a certain continuity. In addition, animal model research related to tumorigenesis needs to cross the gap of species. Therefore, it is more reasonable and scientific to search for early events that drive liver cancer in a series of samples with the same background. We recruited 5 patients who were clinically diagnosed as PLC and were pathologically diagnosed as early liver cancer with liver Dn. The liver fibrosis tissues, Dn tissues and HCC tissues of each patient were taken as a group and detected by methylation chip. Since the genetic backgrounds of different types of samples are the same, the heterogeneity within the group is controlled to a minimum, which can better clarify the sequential changes of epigenetic modifications during the "inflammatory cancer transformation" process.

Overall, our study systematically reveals the dynamic changes of the DNA methylation in the early stages of liver cancer, finds the DNA methylation sites that drive liver cancer, and clarifies the biological functions and regulatory mechanisms of downstream genes regulated by related DNA methylation sites. Collectively, these results provide the new target and clinical evidence for early diagnosis and precise treatment of liver cancer.

\section{Methods}




\section{Mice in vivo limiting dilution assay and DEN-induced carcinogenesis mice model}

Male mice (nude), 6-8 weeks old and male mice (C57BL/6), 1week old were purchased from Animal Research Center of Naval Medical University. For mice in vivo limiting dilution assay, the 48 nude mice were divided into 8 groups, 6 in each group. Nude mice in each group were injected under the axilla of the right forelimb with the ZNF334-OE cells or its control cells (ZNF334-NC) at $5 \times 10^{6}, 1 \times 10^{6}, 5 \times 10^{5}, 1 \times 10^{5}$ cells/group respectively. Subcutaneous tumors of the mice were collected after two months. The number and size of tumors were recorded. The website ELDA (http://bioinf.wehi.edu.au/software/elda/) was used to analyze the proportion of cancer stem cells in the mouse subcutaneous tumors and evaluate its sphere formation ability. For DEN-induced carcinogenesis mice model, the $30 \mathrm{C} 57 \mathrm{BL} / 6$ mice were divided into 2 groups, 15 in each group. The chemical HCC was induced by the combination of DEN $(25 \mathrm{mg} / \mathrm{kg}$ i.p.) given at week 2 postpartum followed by weekly injections of $\mathrm{CCl}_{4}(0.5 \mathrm{~mL} / \mathrm{kg}$ i.p., dissolved in olive oil). Mice were sacrificed at the indicated time for further studies. All animal experiments were approved by the Animal Care Committee of Naval Medical University, and the investigation complied with the Guide for the Care and Use of Laboratory Animals of the U.S. National Institutes of Health.

\section{Human liver tissue}

A total of 213 specimens of patients with HCC from September 2012 to September 2019 were selected from the liver tissue specimen bank of Eastern Hepatobiliary Surgery Hospital, of which 5 cases had HBV infection background and were clinically diagnosed as primary liver cancer and pathologically diagnosed as early liver cancer with liver dysplasia nodules. HCC tumor tissues (the site where the tumor grows vigorously) and the matched adjacent tissues (more than $2 \mathrm{~cm}$ from the tumor margin) were collected. All patients who provided liver samples signed an informed consent form before the operation and reported to the hospital ethics committee for approval.

\section{Cell culture and experimental conditions}

Human HCC cell lines (Huh7, HepG2, Hep3B, Sk-Hep1, SNU387) and normal liver cell lines (CCC-HEL-1, L02) were purchased from Chinese Academy of Sciences Cell Bank. The above-mentioned cells were cultured in a high-sugar DMEM medium containing $10 \% \mathrm{FBS}$ at $37^{\circ} \mathrm{C}$ and $5 \% \mathrm{CO}_{2}$.

\section{Detection and analysis of DNA methylation}

The liver fibrosis tissues, dysplastic nodule tissues, and early liver cancer tissues of 5 patients were extracted with genomic DNA by QIAamp DNA Kit (QIAGEN), and then treated with sulfite to make unmethylated $\mathrm{C}$ into $\mathrm{U}$, and the methylated $\mathrm{C}$ remains unchanged. DNA sample quality inspection and methylation microarray detection were carried out in accordance with the standard procedures of Infinium ${ }^{\circledR}$ Methylation EPIC. R package 'RnBeads' was used to analyze microarray data and identify differentially methylated sites $(\beta>0.1$ and $p<0.01)$. Principal component analysis, k-means clustering analysis, and heatmap of DNA methylation levels were performed through R platform. Gene function enrichment analysis were conducted using GSEA (gene set enrichment analysis). 


\section{MassArray methylation quantitative detection}

MassARRAY methylation detection technology (Sequenom, USA) performed by CapitalBio Technology (Shanghai, China) was used to detect the methylation level of ZNF334 promoter (human) in carcinoma and paracancerous tissues of $25 \mathrm{HCC}$ patients and Zfp334 promoter (mice) in treatment and control group. For mice Zfp334 promoter, three primers were designed to cover all the $\mathrm{CpGs}$. The primers are as follows:

\begin{tabular}{|ll|}
\hline Primer names & Sequences \\
\hline ZNF334-5\#-F & aggaagagagGGTTAGGAGTTTAATTTTGTTTTGGT \\
\hline ZNF334-5\#-R & cagtaatacgactcactatagggagaaggctAACTCCTCAAAAATCCCTCAAAATA \\
\hline Zfp334-6\#-F & aggaagagagGGGTTTTTTGGGAGTTATAAAAGAA \\
\hline Zfp334-6\#-R & cagtaatacgactcactatagggagaaggctAATACAAAAACCTTTTTCTCAACCA \\
\hline Zfp334-14\#-F & aggaagagagTTGTTGGTTTGTTTTTTTAGGTTTT \\
\hline Zfp334-14\#-R & cagtaatacgactcactatagggagaaggctAATCTACTTCCCTTACTTTCCCTAA \\
\hline Zfp334-16\#-F & aggaagagagGAAGGTATTGTTAGATGTGTTGGAG \\
\hline Zfp334-16\#-R & cagtaatacgactcactatagggagaaggctAAAAAAAACAACAACAAACACCATT \\
\hline
\end{tabular}

\section{Lentiviral transfection}

A stable ZNF334-overexpressing cell line (ZNF334-OE) and a control cell line (ZNF334-NC) were constructed in the Huh7; A stable ZNF334-knockdown cell line (sh-ZNF334) and its control cell line (shNC) were constructed in the SNU387. The ZNF334 overexpression lentivirus is synthesized and packaged by Gene Pharma (Shanghai, China). Its overexpression vector is EF1a-CMV-GFP-T2A-puro, and the virus titer is $1.0 \mathrm{E}+8$. The ZNF334 knockdown lentivirus was synthesized and packaged by Genechem (Shanghai, China). The shRNA vector was hU6-MCS-Ubiquitin-EGFP-IRES-puromycin, and the virus titer was 1.0E+9. Its interference fragment was 1\#GAGGGCAAUUCUCAUUACATT;

2\#UGUAAUGAGAAUUGCCCUCTT. When the cell confluence is about $60 \%$, virus solution and PolyBrene $(5 \mu \mathrm{g} / \mathrm{ml})$ were added to promote infection. For determination of the infection efficiency, fluorescence microscope was used after $72 \mathrm{~h}$, and the cells were selected with puromycin.

\section{Dual-Luciferase reporter assay}

The dual luciferase reporter plasmid psiCHECKTM-2 was synthesized by Gene Pharma (Shanghai, China). The promoter region of ZNF334 is defined as the transcription start site to the upstream 2000bp. Dual-Luciferase Reporter Assay System (Promega, E1910) for dual-luciferase reporter gene detection was used according to the user manual. The 293T cells that had been transfected with dual fluorescence and 
transcription factor plasmids or control plasmids were lysed with lysis buffer, and then firefly fluorescence and renilla fluorescence values were detected in a microplate reader, and statistical analysis was performed to compare the differences between the groups. The primer sequence of ZNF334 promoters are as follows:

\begin{tabular}{|ll|}
\hline ZNF334 Primer names & Sequences \\
\hline promoter-2.0k-F & CATGGCTCGACAGATCTTGTTCCCAATTACAAAAG \\
\hline promoter-2.0k-R & TTGGAAGCCATGGTGGCTAGCTGGCGAACCGGAAGGGCG \\
\hline promoter-1.5k-F & CATGGCTCGACAGATCTTGTTCCCAATTACAAAAG \\
\hline promoter-1.5k-R & TTGGAAGCCATGGTGGCTAGCGGAACTCATATAGGTCTAG \\
\hline promoter-1.0k-F & CATGGCTCGACAGATCTTGTTCCCAATTACAAAAG \\
\hline promoter-1.0k-R & TTGGAAGCCATGGTGGCTAGCGAATAAATAGAA CTTTA \\
\hline promoter-0.5k-F & CATGGCTCGACAGATCTTGTTCCCAATTACAAAAG \\
\hline promoter-0.5k-R & TTGGAAGCCATGGTGGCTAGCAGTTCCTGGATGACTTCAG \\
\hline
\end{tabular}

\section{Chromatin immunoprecipitation assay}

SimpleChIP® Enzymatic Chromatin IP Kit (CST,Magnetic Beads囚\#9003) was used according to the user manual. Cells were cross-linked with $35 \%$ formaldehyde, immunoprecipitated, de-cross-linked, and DNA purified, and then subjected to qRT-PCR detection. The antibodies used are as follows: Anti-P53 antibody (Active Motif, \# AB_2793254). The primers are as follows:

\begin{tabular}{|lll|}
\hline Primer names & Sequences & \\
\cline { 2 - 3 } & Forward (5' 3') $^{\prime}$ & Reverse (5' 3') $^{\prime}$ \\
\hline ZNF-P53-1 & TCTCTTTTTCCATGTGGTCTC & TTTGCCCTTTGAAGAGTCCTT \\
\hline ZNF-P53-2 & AAGTCAACGTGGGAGGGAAA & ATTCCAGAAAAGAACAATTAG \\
\hline ZNF-P53-3 & GTACGTTTACTGCACCTTCCC & ACAGGCCTGTGTGGCATGTCA \\
\hline ZNF-P53-4 & GTTACCATTGCATATGGTATTC & TCTAGACAATATAGTCTACTAC \\
\hline ZNF-P53-5 & AATAACAAATTACTAGACCTATA & TCAGAATATCTCTGAAGAAA \\
\hline ZNF-P53-6 & TTCTGGGGACTGTGGTCCGGAA & GTCCAGGTAAAAAACAGGA \\
\hline Note: The amplified sequence of primer ZNF-P53-6 contains the cg07139762 site of the EPIC chip \\
\hline
\end{tabular}




\section{Flow Cytometry}

Annexin V: FITC Cell Apoptosis Detection Kit (BD, \#556547) was used to determinate the cell apoptosis. Cells were collected, blocked with non-specific antigen, incubated with direct-labeled flow cytometry antibody, and then performed in BD LSR】.

\section{Cell apoptosis detection (TUNEL)}

Cells were seeded on the slide of 6 -well plates. When the cell confluence is about $50 \%$, for determination of cell apoptosis, a TUNEL Apoptosis Detection Kit (Beyotime, C1086) was used according to the user manual. The slides were fixed, stained and observed under a microscope to obtain images.

\section{Cell proliferation assay (CCK8 and EdU)}

Cells were seeded $(2,000 /$ well $)$ and maintained in 96 -well plates. For determination of cell proliferation at indicated time points $(0,12,24,48,60$ and $72 \mathrm{~h}$ after adherence), a Cell-Counting Kit 8 (Beyotime,C0038) was used according to the user manual. Fluorescence $(450 \mathrm{~nm})$ was recorded in a microplate reader.

Cells were seeded on the slide of 6-well plates. When the cell confluence is about $50 \%$, for determination of cell proliferation, a Cell-Light EdU Apollo643 In Vitro Kit (100T) (RIBOBIO, C10310-2) was used according to the user manual. The slides were fixed, stained and observed under a microscope to obtain images.

\section{Quantitative real-time PCR}

Total RNA of the cells was extracted with the Trizol (Invitrogen), and the cDNA synthesis was completed with the TAKARA reverse transcription kit (TAKARA0360A). Roche LightCycler 96 was used for qRT-PCR experiments. The primers are as follows:

\begin{tabular}{|lll|}
\hline Gene & Forward $\left(\mathbf{5}^{\prime} \rightarrow \mathbf{3}^{\prime}\right)$ & Reverse $\left(\mathbf{5}^{\prime} \rightarrow \mathbf{3}^{\prime}\right)$ \\
\hline ZNF334 & AGGGGAGACAGACTGAAAGGA & GTGAGGCTTGTCTTCACACG \\
\hline GAPDH & AGCGAGCATCCCCCAAAGTT & GGGCACGAAGGCTCATCATT \\
\hline ACTB & CCACCATGTACCCTGGCATTG & TCATCTTGTTTTCTGCGCAAGTTA \\
\hline EpCAM & AATCGTCAATGCCAGTGTACTT & TCTCATCGCAGTCAGGATCATAA \\
\hline CD133 & AGTCGGAAACTGGCAGATAGC & GGTAGTGTTGTACTGGGCCAAT \\
\hline CD90 & ATCGCTCTCCTGCTAACAGTC & CTCGTACTGGATGGGTGAACT \\
\hline CTS & GGAGAGGGATTTGCAGGTGTA & CATTGTGGGCAAGGTGCTATT \\
\hline CD24 & CTCCTACCCACGCAGATTTATTC & AGAGTGAGACCACGAAGAGAC \\
\hline
\end{tabular}




\section{Western blot}

Cells were lysed with RIPA Lysis Buffer (Beyotime, P0013C) to obtain total protein and then quantified by BCA method, and then $20 \mu \mathrm{g}$ of each sample was subjected to SDS-PAGE electrophoresis and transferred to PVDF membrane for exposure. The antibodies used are as follows: Anti-ZNF334 (Abcam, \#ab127712), Anti-GAPDH (Abcam, \# ab8245), IRDye800CW goat anti-rabbit IgG (Licor, \#926-3221), IRDye 680LT goat anti-mouse lgG (Licor, \#926-68020).

\section{Plate clone formation assay}

Cells were seeded (2,000/well) and maintained in 6-well plates with FBS-free medium for two weeks and then fixed with formaldehyde, stained with crystal violet. The number of cell clusters in each well was counted and photographed.

\section{Sphere formation assay}

Cells were seeded (2,000/well) and maintained in low-attachment 6-well plates with FBS-free medium for two weeks. The number of spheroids and the size of cell clumps, were recorded under a microscope.

\section{Extra limiting dilution assay}

Cells were resuspended and seeded $(100,200,400,800$ /well for $48,24,12,6$ wells respectively) in lowattachment 6-well plates with FBS-free medium for two weeks. The number of wells containing spheroids were counted and recorded under a microscope. The website ELDA (http://bioinf.wehi.edu.au/software/elda/) was used to analyze the proportion of cancer stem cells.

\section{Immunofluorescence staining}

Cells were seeded on the slide of 6 -well plates. When the cell confluence is about $60 \%$, primary antibody (Abcam, \#ab127712), secondary antibody (Licor, \#926-3221) and DAPI were used according to the user manual to stain the cells. Confocal laser microscope was used for taking pictures.

\section{Statistical analysis}

All the data were expressed as mean \pm SD for at least three independent experiments and were analyzed by two-sided Student's t test, one-way ANOVA or two-way ANOVA. Non-parametric test was used when data does not meet the normal distribution and homogeneity of variance. Log rank test and Cox regression model were used in the survival analysis. The survival curve was drawn using the KaplanMeier method. All statistical analysis was performed using GraphPad Prism 7.0 and relevant R packages. Probability $(\mathrm{P})$ values $\leq 0.05$ were considered to be statistically significant. ${ }^{*}, p<0.05 ; * \star, p<0.01 ; * \star \star, p<$ $0.001 ; * \star * *, p<0.0001$.

\section{Results}




\section{Hypermethylation of TSGs occurs in the early stage of HCC}

In the study, we recruited five liver cancer patients. Specially, we obtained cirrhosis, liver dysplastic nodules (dysplasia) and early HCC tissues for each patient (Figure 1A). DNA methylation was measured in these fifteen liver tissues. Analyzing DNA methylation profiles, we obtained the methylation levels of all $23638 \mathrm{CpG}$ sites across the whole genome. Principal component analysis indicated that cirrhosis, dysplasia and HCC have very distinct methylation spectrum (Figure 1B). In the first stage from cirrhosis to dysplasia and the second stage from dysplasia to HCC, we separately identified 6808 and 2956 differentially methylated sites (Figure S1D, S1E). Among differentially methylated sites, 3762 (55.25\%) and 635 (21.48\%) are hypermethylated in the first and second stages, respectively (Figure S1C). Analyzing differentially methylated sites, we further identified 108 (18.62\%) hypermethylated and 472 (81.38\%) hypomethylated regions in the stage from cirrhosis to dysplasia (Figure 1C, 1D, S1A, S1B). In the stage from dysplasia to HCC, there are $26(8.23 \%)$ hypermethylated and $290(91.77 \%)$ hypomethylated regions. Most of these differentially methylated regions are from the exonic, intronic and intergenic regions (Figure S1F). Based on all differentially methylated regions, cirrhosis samples were separated from dysplasia and HCC and dysplasia was more similar to HCC (Figure 1E). Next, we identified the genes associated with differentially methylated regions. In the stage from cirrhosis to dysplasia, $76.56 \%$ hypermethylated genes are oncogenes (Figure 1F). In the stage from dysplasia to HCC, the fraction of hypermethylated oncogenes is $82.35 \%$ (Figure 1F). Higher fraction of tumor suppressor genes is hypermethylated but not hypomethylated in both stages. Using K-means clustering, we separated hypermethylated genes into two groups according to their methylation levels (Figure 1G). Function enrichment analysis indicated that the genes with higher methylation levels were significantly regulated by TP53 transcription factors (Figure1H).

\section{Hypermethylation of ZNF334 promoter remains throughout the process of inflammatory-to-cancer transformation in DEN-induced carcinogenesis mice model}

Next, the differentially methylated sites of the EPIC chip were integrated with the p53 protein ChIP-seq data (GSE55727) and p53 DNA binding motif from JASPAR database. The results showed that CELF2 and ZNF334 are the p53 target genes shared by the three data (Figure 2A). Analysis of the relationship between the expression of CELF2 and ZNF334 and patient prognosis showed that CELF2 has no correlation with patient survival (data not shown). In addition, the LIHC cohort in the TCGA database only showed that ZNF334 was associated with patient prognosis (Figure S1F). In order to confirm whether there is hypermethylation in the ZNF334 promoter in the process of inflammatory-to-cancer transformation, we constructed a DEN-induced carcinogenesis mice model (Figure 2B) and examine the DNA methylation level of ZNF334 (also called Zfp334 in mice) promoter. The results showed that compared with the control group, the Zfp334 promoter of the treatment group had higher DNA hypermethylation (Figure 2C, 2D, 2F, S2), and there was no difference in the average degree of methylation at each time point, indicating hypermethylation of Zfp334 promoter goes all through the process of inflammatory-to-cancer transformation in mice liver cancer model (Figure 2E).

\section{Hypermethylation of ZNF334 promoter inhibits the expression of ZNF334 in HCC patients}


Firstly, we found a negative correlation between the ZNF334 methylation level and ZNF334 mRNA expression (Pearson $=-0.53, \mathrm{R}^{2}=0.28, p=4.95 \mathrm{e}-28$; TCGA, Firehose Legacy, 373 samples). For tissues from patients, the expression of ZNF334 at mRNA and protein levels in the tumor tissues significantly decreased by detecting the cancer and adjacent tissues of 213 liver cancer patients (Figure 3B,C). In addition, methylation mass detection primers (Figure 3D) for the ZNF334 promoter region (the 2000bp region upstream of the transcription start site, TSS) were designed, and used in the DNA methylation detection in cancer and adjacent tissues of 25 liver cancer patients in our hospital. The results of MassARRAY methylation detection showed that the degree of methylation in the ZNF334 promoter region of cancer and adjacent tissues of the same patient was significantly different, and the degree of methylation of cancer tissue was significantly higher than that of adjacent tissues (Figure 3E, F, G).

\section{Transcription factor p53 can regulate the expression of TSG ZNF334}

In order to further investigate the relationship between p53 and ZNF334, we used the dual luciferase reporter plasmid system to construct the full length of the promoter region of ZNF334 (2000bp DNA fragment, $1500-2000 \mathrm{bp}$ upstream of the TSS containing the EPIC chip probe cg07139762) into the plasmids expressing dual luciferase (Figure 4A up). The results of the dual luciferase reporter experiment showed that, compared with the control plasmid, the plasmids co-transfected with p53 and ZNF334 can significantly increase the expression abundance of luciferase in SNU387, which demonstrated that p53 can bind to the promoter region of ZNF334 and promote its transcription. The promoter region of ZNF334 was further truncated (Figure 4A down) for dual luciferase reporter gene experiments. The results showed that ZNF334 promoters with $1500 \mathrm{bp}$ or $1000 \mathrm{bp}$ length can still bind to $\mathrm{p53}$, but the luciferase did not express when ZNF334 promoter region was truncated to 500bp length (Figure 4B). Therefore, we predict that there may be multiple binding sites between p53 and ZNF334 promoter ranging from 500 to $2000 \mathrm{bp}$ upstream of the TSS. In order to further verify whether the p53 binds to the ZNF334 promoter, we used JASPAR (transcription factor binding site prediction website) to predict the binding sites between p53 and ZNF334 promoter (Figure 4C), where the $\mathrm{p} 53$ binding site sequence (GGGGGCCTTTCTGCGCATGT) contains the EPIC chip probe cg07139762. Subsequently, primers were designed for the predicted sites to perform chromatin immunoprecipitation (ChIP) experiments. The results of ChIP experiments showed that in Huh7 cell, the binding of p53 to the ZNF334 promoter region was significantly higher than that of IgG, which proved that p53 can bind to the ZNF334 promoter region (Figure 4D,E).

\section{ZNF334 can promote liver cancer cell apoptosis, inhibit the proliferation and stemness of liver cancer cells in vitro}

To further clarify the biological feature of ZNF334 in HCC, the expression of ZNF334 in different HCC cell lines (Huh7, HepG2, Hep3B, Sk-Hep1, SNU387) and normal liver cell lines (CCC-HEL-1, L02) were measured. The results of qRT-PCR and western blot both showed that ZNF334 expressed lower in Huh7, and higher in SK-Hep1, SNU387 and CCC-HEL-1 (Figure 5A,C). Therefore, based on the expression level of ZNF334 in different cells, we used lentiviral vectors to construct a stable transgenic Huh7 cell line overexpressing ZNF334 (ZNF334-OE) and its control cell line (ZNF334-NC); a cell line of which ZNF334 was stably knocked down (sh-ZNF334) and its control cell line (sh-NC) were constructed using SNU387 
cell line (Figure 5B,D). The results of immunofluorescence showed that in HepG2 and SNU387, ZNF334 was expressed to varying degrees in the nucleus and cytoplasm (Figure $5 \mathrm{E}$ ). The cell proliferation assay (CCK8) showed that compared with ZNF334-NC, the proliferation ability of ZNF334-OE was significantly reduced (Figure 5F), while improved in sh-ZNF334 (Figure 5G). Similarly, the proliferation rate of ZNF334OE marked with EdU was much lower than that of the control group in the case of seeding the same number of cells (Figure $5 \mathrm{~J}, \mathrm{H}$ ). In addition, plate clone formation assay also showed that compared with ZNF334-NC, fewer tumor clumps with smaller size were formed in ZNF334-OE, while the clone formation ability of sh-ZNF334 was significantly higher than sh-NC, which further proved that overexpression of ZNF334 can inhibit the proliferation of tumor cells (Figure 5M). Besides, we also found that the proportion of ZNF334-OE undergoing apoptosis increased significantly when the same number of cells were inoculated (Figure $5 \mathrm{~L}, \mathrm{I}$ ). And compared with the control group, the apoptosis of ZNF334-OE was mainly due to the increase in early apoptosis level (Figure 5K). We further evaluated the effects of different expression levels of ZNF334 on the stemness of HCC cell lines. The results of the sphere formation assay showed that the number of cell clumps formed by ZNF334-OE decreased, while the spheronization ability of sh-ZNF334 cells was significantly higher than that of sh-NC (Figure $5 \mathrm{~N}$ ). Limiting dilution assay (LDA) in vitro showed that the proportion of ZNF334-OE tumor stem cells was significantly lower than that of the control group, while the number of cell clumps formed by sh-ZNF334 was significantly higher than that of the control group, further indicating that overexpression of ZNF334 can reduce the stemness of tumor cells (Figure 6B).

\section{ZNF334 can inhibit tumor formation in vivo}

Limiting dilution assay in vivo showed that under the condition of injecting the same number of tumor cells, the number of tumors formed under the skin of mice with ZNF334-NC injected is more than that of the ZNF334-OE group, and a certain number of tumors can still be formed at a low concentration $\left(5 \times 10^{5}\right)$. On the other hand, fewer subcutaneous tumors were formed in the mice with ZNF334-OE injected, and even no tumor formed at $1 \times 10^{5}$ concentration (Figure 6A). Using Extreme Limiting Dilution Analysis (ELDA, a website analysis tool), we found that the proportion of stem cells in ZNF334-OE cells was significantly lower than that of the control group (Figure 6C, D). Further measuring the markers related to the stemness of tumor cells in tumor tissues of the mice, we found that the expression of stemness markers such as EpCAM, CD13, CD24, CD44, CD90 and CD133 in ZNF334-OE cells was significantly lower than that of the control group, which was consistent to the phenotype of the mice model (Figure 6E).

\section{The overall survival and relapse-free survival of patients with high expression of ZNF334 are longer}

In order to further investigate the relationship between ZNF334 and the prognosis of liver cancer patients, we collected the tumor tissues from 213 liver cancer patients and detected the expression of ZNF334 at the RNA level. According to Youden index $\left(\mathrm{OS}_{\text {Youden index }}=4.152, \mathrm{RFS}_{\text {Youden index }}=4.088\right.$, Figure $\left.7 \mathrm{G}, \mathrm{H}\right)$, patients were divided into ZNF334 high expression group $(n=107)$ and low expression group $(n=106)$. Chi-square showed that ZNF334 is related to tumor growth-related indicators-alpha-fetoprotein (AFP), number of tumors and tumor capsule, and is also concerned with metastasis-related indicators-portal vein tumor thrombus. Compared with the ZNF334 high expression group, the proportion of people with 
high serum $\mathrm{AFP}(>400 \mathrm{ng} / \mathrm{ml})$,tumor number and portal vein tumor thrombus is greater, while the proportion of people with tumor capsule is lower. These results suggest that the ZNF334 level is negatively correlated with the malignancy of liver cancer (Supplementary table 1).

Cox univariate analysis found that ZNF334, AFP, AST, and MVI were related to overall survival (OS) in patients with liver cancer (Figure 7A); ZNF334, AFP, MVI, and portal vein tumor thrombus were related to postoperative relapse-free survival (RFS) (Figure 7A,B). Cox multivariate analysis found that AFP > $400 \mathrm{ng} / \mathrm{ml}$, low ZNF334 expression and MVI are independent risk factors that affect the patients' OS (Figure 7C); while AFP > 400ng/ml, low ZNF334 expression and portal vein tumor thrombus are the independent risk factors that affect patients' RFS (Figure 7D). OS and RFS Kaplan-Meier Curve showed that the OS and RFS of the ZNF334 high expression group were higher than those of the low expression group (pOS< 0.001; pRFS <0.001) (Figure 7E, F).

\section{Discussion}

Studies have shown that changes in genomic epigenetics are closely related to the occurrence of cancer[20-22]. In this study, the EPIC methylation chip was used to systematically detect the DNA methylation conditions in fibrosis tissues, Dn tissues and early hepatocarcinoma tissues of the same patient. The results showed that the DNA methylation lineages in the three tissues were significantly different. In the early stage of liver cancer (stage 1), the proportion of DNA hypermethylation modifications was higher than that of hypomethylation modifications, and the relevant sites were mainly concentrated in TSGs, which suggests that the hypermethylation modification of TSGs may be the main epigenetic feature in the early stage of liver cancer, promoting the occurrence of liver cancer to a certain extent. Studies have reported that in tumor tissues, the increase in hypermethylation of TSGs and the decrease in methylation of the whole genome are primary causes of cancer[23-27], which is consistent with our results.

As an important transcription factor in the body, p53 participates in regulating cell growth, maintaining genome stability, and inhibiting tumor angiogenesis[28]. As a tumor suppressor gene, wild-type p53 can inhibit the occurrence of tumors[29], while mutant p53 affects the regulation level of downstream molecules and promotes tumorigenesis. Studies have shown that mutant p53 exists in over $50 \%$ of tumor tissues[30], contributing to the occurrence and development of tumors[31]. In this study, k-means clustering analysis and gene function enrichment analysis were performed on the DNA hypermethylation modified regions in the stage 1 process, and it was found that the target gene of p53 was significantly enriched in the DNA hypermethylation segment.

Additionally, studies have shown that the DNA methylation modifications can affect the transcription of TSGs. Variations in DNA methylation modification status will cause changes in the binding of the key transcription factors to downstream TSGs[32]. When the promoter of a TSG is modified by hypermethylation, the transcription factor cannot combine with it, and lead to the silence of the gene with a decrease in its transcription[33-36], which is recognized as the upstream molecular mechanism of 
some TSGs losing their biological functions. In this study, we compared the differential methylation site data with the relevant data in the existing database, and screened out the target gene ZNF334 downstream of $\mathrm{p} 53$. And in order to further verify the regulation mechanism of the transcription factor p53 on the gene ZNF334, we performed the dual luciferase reporter gene experiment and ChIP experiment to prove that the p53 can regulate the expression of ZNF334 by binding to the ZNF334 promoter. And meanwhile, in clinical samples, we used MassArray Sequenom detection to further verify the DNA methylation changes of ZNF334 promoter in cancerous tissues and adjacent tissues. The results found that in tumor tissues, the degree of hypermethylation modification in the ZNF334 promoter increased, which may be the main reason for the decline binding of p53.

In order to further explore the biological role of ZNF334 in the development of liver cancer, a series of in vivo and in vitro experiments were designed to explore the function of ZNF334. The results showed that ZNF334 can significantly promote the apoptosis of liver cancer cells, inhibit the proliferation and stemness of liver cancer cells in vitro, and can significantly inhibit the occurrence of tumors in the subcutaneous tumor-bearing mouse model. In addition, combined with clinical samples and follow-up data, we found that compared with paracancerous tissues, the expression of ZNF334 in tumor tissues of liver cancer patients was significantly reduced. Patients with high ZNF334 expression had higher recurrence-free survival and overall survival rates than those with low expression.

As we all know, ZNF334 is a member of the zinc finger protein family and our immunofluorescence results showed that ZNF334 is expressed in both nucleus and cytoplasm. Hence, we guess that ZNF334 is likely to function as a transcription factor and bind to some downstream genes' promoters, thus affecting their expression; it may also work as a secreted protein or intracellular protein to interact with other proteins in the cell and affect its function. Therefore, the molecular mechanism downstream of ZNF334 needs further research and confirmation.

In conclusion, our study constructed a sequential map of DNA methylation modification during the occurrence of hepatocellular carcinoma, clarified the temporal and spatial characteristics of DNA hypermethylation modification and firstly identified the regulatory mechanism of p53 on its downstream target gene ZNF334. Furthermore, we explored the biological role of tumor suppressor gene ZNF334 in the occurrence of liver cancer and provided a new target and clinical evidence for the early diagnosis and precise treatment of liver cancer.

\section{Conclusions}

We constructed a sequential map of DNA methylation modification during the occurrence of HCC, and clarified the biological function and regulatory mechanism of the tumor suppressor gene ZNF334, which is regulated by related DNA methylation sites, and also provide new targets and clinical evidence for the early diagnosis and precise treatment of liver cancer.

\section{List Of Abbreviations}


ZNF334, Zinc Finger Protein 334; HCC, Hepatocellular carcinoma; PLC, Primary liver cancer; TSGs, Tumor suppressor genes; Dn, Dysplastic nodules; TSS, Transcription start sites; OS, Overall survival; RFS, Recurrence-free survival rate; TNF-a, Tumor necrosis factor-a; ROC, Receiver operating characteristic curve; HBV, Hepatitis virus B; ALT, Alanine aminotransferase; MVI, Microvascular invasion; TACE, Transcatheter arterial chemoembolization; GSEA ,Gene set enrichment analysis; ELDA, Extreme Limiting Dilution Analysis; LDA, Limit dilution assay.

\section{Declarations}

\section{Ethical approval and consent to participate}

This study was approved by the Research Ethics Committee of the third affiliated hospital of Naval Medical University. And all patients have assigned the informed consents.

\section{Consent for publication}

Not applicable

\section{Availability of data and materials}

The datasets used and analyzed during the current study are available from the corresponding author on reasonable request.

\section{Competing interests}

All authors who have taken part in this study reported no conflicts of interest. The authors are responsible for the content.

\section{Funding}

This work was supported by Shanghai Rising-Star Program (19QA1408700, 20QA1412000)هNational Key Research and Development Program of China (2016YFC1302303); State key infection disease project of China (2018ZX10732202-002-005); National Natural Science Foundation of China (81972575, 81972657, $81672345,81802983,81702734,82073031 \varangle 82002458$ ); Shanghai Sailing Program (19YF1459600) \the San Hang Program of Naval Medical University $\llbracket$ Meng Chao's talent training program for young doctors.

\section{Author Contribution}

WPZ and SXY formulated the research concept and designed the study, FY and JW supervised the study, $\mathrm{LL}$ and YY collected the clinical samples, DPS carried out the research, XJG analyzed the clinical data, WL, WBD, DYD analyzed the pathological characteristics. LHZ and GJH validated the results, JYJ and JY performed the formal analysis and software implementation, DPS wrote the original draft ,XJG, LL and YY revised and edited the draft. All authors have read and approved the final manuscript. 
Acknowledgements

Not applicable.

\section{References}

1. Kim E, Viatour P. Hepatocellular carcinoma: old friends and new tricks. Exp Mol Med 2020;52:1898907.

2. Kulik L, El-Serag HB. Epidemiology and Management of Hepatocellular Carcinoma. Gastroenterology 2019;156:477-91 e1.

3. Chen W, Zheng R, Baade PD, et al. Cancer statistics in China, 2015. CA Cancer J Clin 2016;66:115-32.

4. Fu J, Wang H. Precision diagnosis and treatment of liver cancer in China. Cancer Lett 2018;412:2838.

5. Sia D, Villanueva A, Friedman SL, et al. Liver Cancer Cell of Origin, Molecular Class, and Effects on Patient Prognosis. Gastroenterology 2017;152:745-61.

6. Zhou M, Wang H, Zeng X, et al. Mortality, morbidity, and risk factors in China and its provinces, 19902017: a systematic analysis for the Global Burden of Disease Study 2017. Lancet 2019;394:1145-58.

7. Hanahan D, Weinberg RA. Hallmarks of cancer: the next generation. Cell 2011;144:646-74.

8. Lee M, Kim K, Kim SY, et al. Genomic structures of dysplastic nodule and concurrent hepatocellular carcinoma. Hum Pathol 2018;81:37-46.

9. Kojiro M. Premalignant lesions of hepatocellular carcinoma: pathologic viewpoint. J Hepatobiliary Pancreat Surg 2000;7:535-41.

10. Sciarra A, Di Tommaso L, Nakano M, et al. Morphophenotypic changes in human multistep hepatocarcinogenesis with translational implications. J Hepatol 2016;64:87-93.

11. Rajan PK, Udoh UA, Sanabria JD, et al. The Role of Histone Acetylation-/Methylation-Mediated Apoptotic Gene Regulation in Hepatocellular Carcinoma. Int J Mol Sci 2020;21.

12. Toh TB, Lim JJ, Chow EK. Epigenetics of hepatocellular carcinoma. Clin Transl Med 2019;8:13.

13. Hlady RA, Sathyanarayan A, Thompson JJ, et al. Integrating the Epigenome to Identify Drivers of Hepatocellular Carcinoma. Hepatology 2019;69:639-52.

14. Schubeler D. Function and information content of DNA methylation. Nature 2015;517:321-6.

15. Xiong L, Wu F, Wu Q, et al. Aberrant enhancer hypomethylation contributes to hepatic carcinogenesis through global transcriptional reprogramming. Nat Commun 2019;10:335.

16. Khan FS, Ali I, Afridi UK, et al. Epigenetic mechanisms regulating the development of hepatocellular carcinoma and their promise for therapeutics. Hepatol Int 2017;11:45-53.

17. Um TH, Kim H, Oh BK, et al. Aberrant $\mathrm{CpG}$ island hypermethylation in dysplastic nodules and early HCC of hepatitis B virus-related human multistep hepatocarcinogenesis. J Hepatol 2011;54:939-47.

18. Kgatle MM, Setshedi M, Hairwadzi HN. Hepatoepigenetic Alterations in Viral and Nonviral-Induced Hepatocellular Carcinoma. Biomed Res Int 2016;2016:3956485. 
19. Kuramoto J, Arai E, Tian Y, et al. Genome-wide DNA methylation analysis during non-alcoholic steatohepatitis-related multistage hepatocarcinogenesis: comparison with hepatitis virus-related carcinogenesis. Carcinogenesis 2017;38:261-70.

20. Portela A, Esteller M. Epigenetic modifications and human disease. Nat Biotechnol 2010;28:1057-68.

21. Sawan $C$, Vaissiere T, Murr R, et al. Epigenetic drivers and genetic passengers on the road to cancer. Mutat Res 2008;642:1-13.

22. Hatziapostolou M, lliopoulos D. Epigenetic aberrations during oncogenesis. Cell Mol Life Sci 2011;68:1681-702.

23. Baylin SB, Ohm JE. Epigenetic gene silencing in cancer - a mechanism for early oncogenic pathway addiction? Nat Rev Cancer 2006;6:107-16.

24. Rodriguez-Paredes M, Esteller M. Cancer epigenetics reaches mainstream oncology. Nat Med 2011;17:330-9.

25. Huntsman DG, Ladanyi M. The molecular pathology of cancer: from pan-genomics to postgenomics. J Pathol 2018;244:509-11.

26. Sincic N, Herceg Z. DNA methylation and cancer: ghosts and angels above the genes. Curr Opin Oncol 2011;23:69-76.

27. Irizarry RA, Ladd-Acosta C, Wen B, et al. The human colon cancer methylome shows similar hypoand hypermethylation at conserved tissue-specific CpG island shores. Nat Genet 2009;41:178-86.

28. Levine AJ. p53: 800 million years of evolution and 40 years of discovery. Nat Rev Cancer 2020;20:471-80.

29. Boutelle AM, Attardi LD. p53 and Tumor Suppression: It Takes a Network. Trends Cell Biol 2021;31:298-310.

30. Duffy MJ, Synnott NC, O'Grady S, et al. Targeting p53 for the treatment of cancer. Semin Cancer Biol 2020.

31. Huang J. Current developments of targeting the p53 signaling pathway for cancer treatment. Pharmacol Ther 2021;220:107720.

32. Herman JG, Baylin SB. Gene silencing in cancer in association with promoter hypermethylation. N Engl J Med 2003;349:2042-54.

33. Bird AP, Wolffe AP. Methylation-induced repression--belts, braces, and chromatin. Cell 1999;99:451-4.

34. Esteller M. CpG island hypermethylation and tumor suppressor genes: a booming present, a brighter future. Oncogene 2002;21:5427-40.

35. Esteller M. Epigenetic gene silencing in cancer: the DNA hypermethylome. Hum Mol Genet 2007;16 Spec No 1:R50-9.

36. Weber M, Hellmann I, Stadler MB, et al. Distribution, silencing potential and evolutionary impact of promoter DNA methylation in the human genome. Nat Genet 2007;39:457-66.

\section{Figures}


A

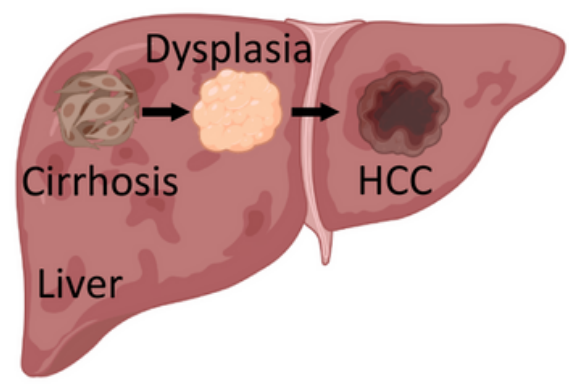

B

$\mathrm{D}$
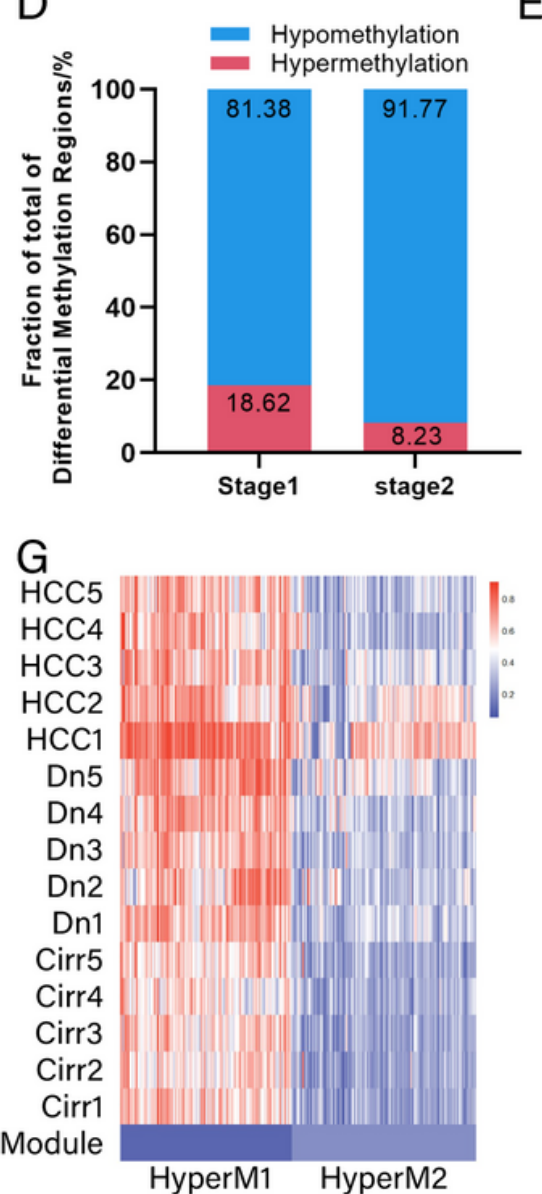

E
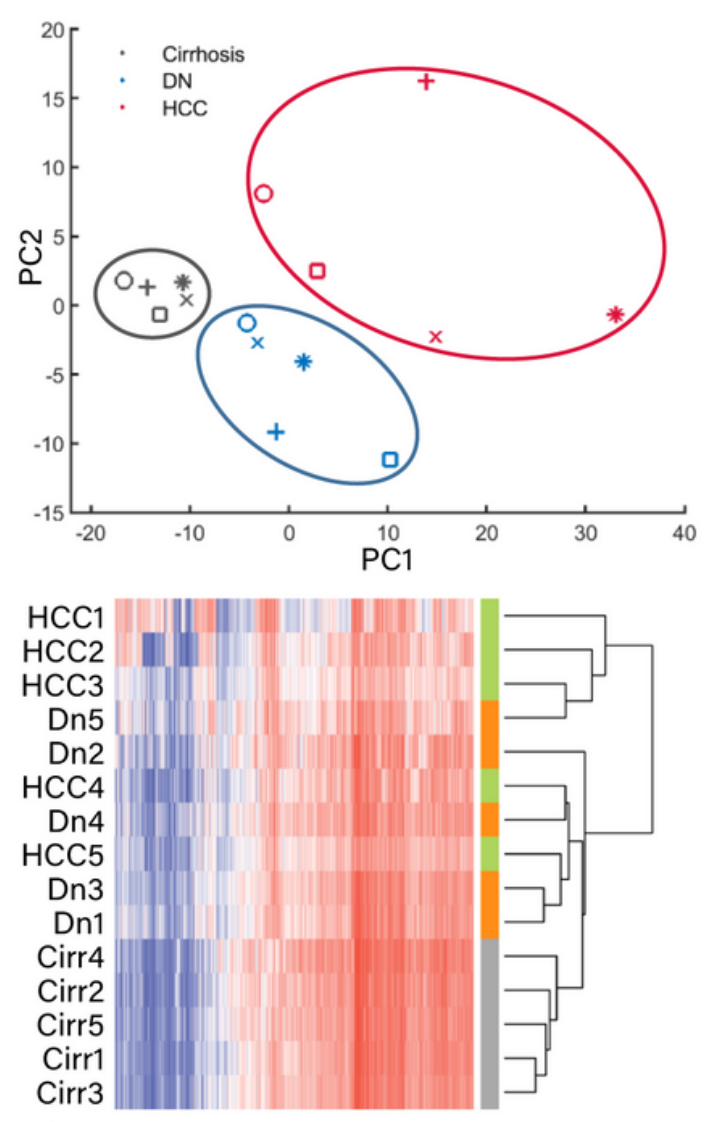

$\mathrm{H}$

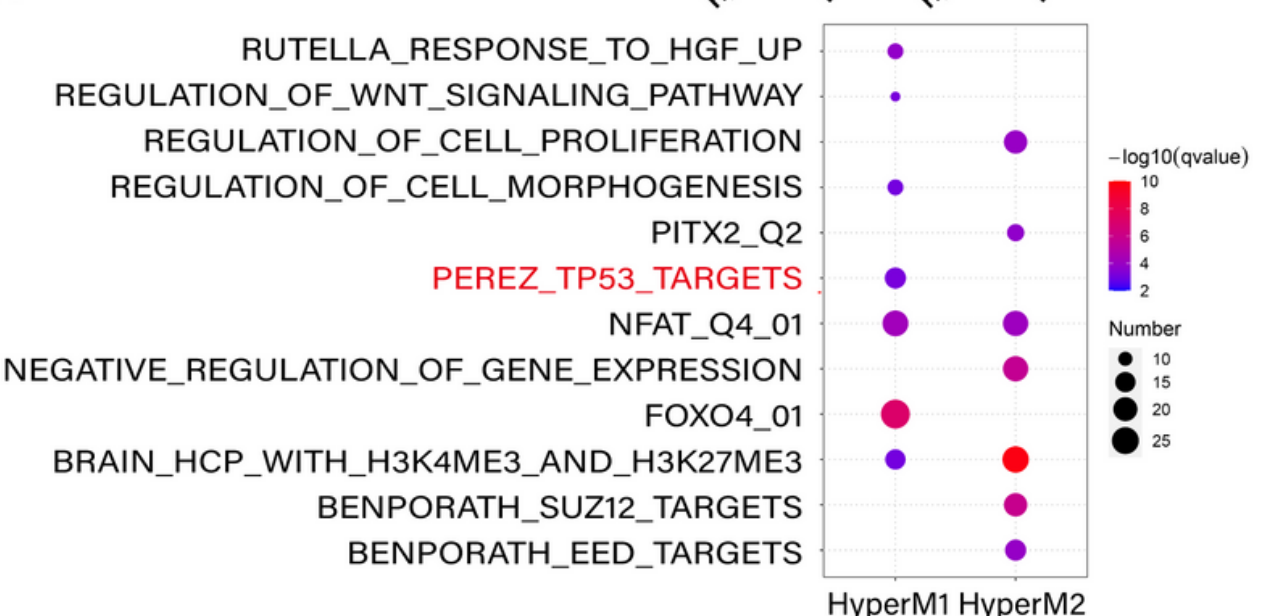

HyperM1 HyperM2

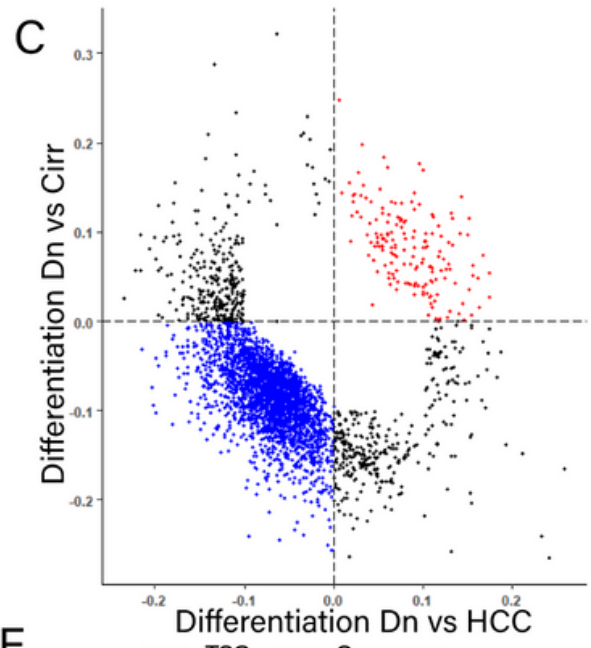

$\mathrm{F}$

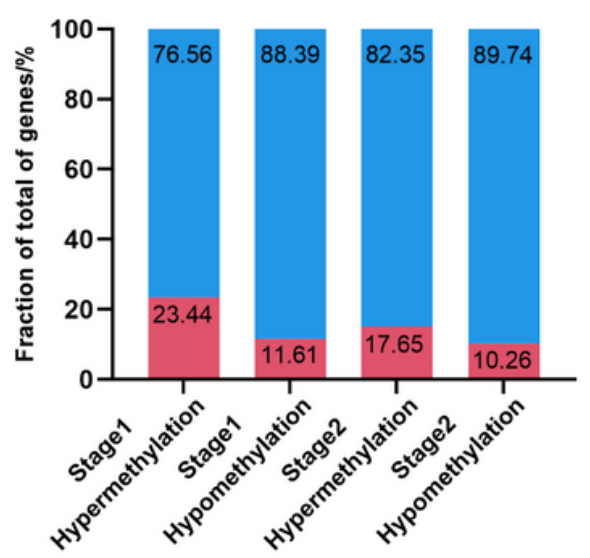

Figure 1

DNA methylation analysis reveals epigenetic changes in the early stage of HCC. A. A diagram of cirrhosis, dysplasia and HCC from each patient. B. Principal component analysis (PCA) of cirrhosis, dysplasia and HCC tissues. PC1 and PC2 indicate principal component 1 and 2, respectively. C. Comparison of differentially methylated regions in the first stage from cirrhosis to dysplasia and the second stage from dysplasia to HCC. D. Fraction of differentially methylated regions in the first and second stages of liver tumorigenesis. E. Clustering analysis of cirrhosis, dysplasia and HCC based on differentially methylated sites. F. Fraction of oncogenes and tumor suppressor genes (TSG) in differentially methylated sites in the 
first and second stages. G. K-means clustering of hypermethylated regions. H. Gene function enrichment analysis of hypermethylated regions.
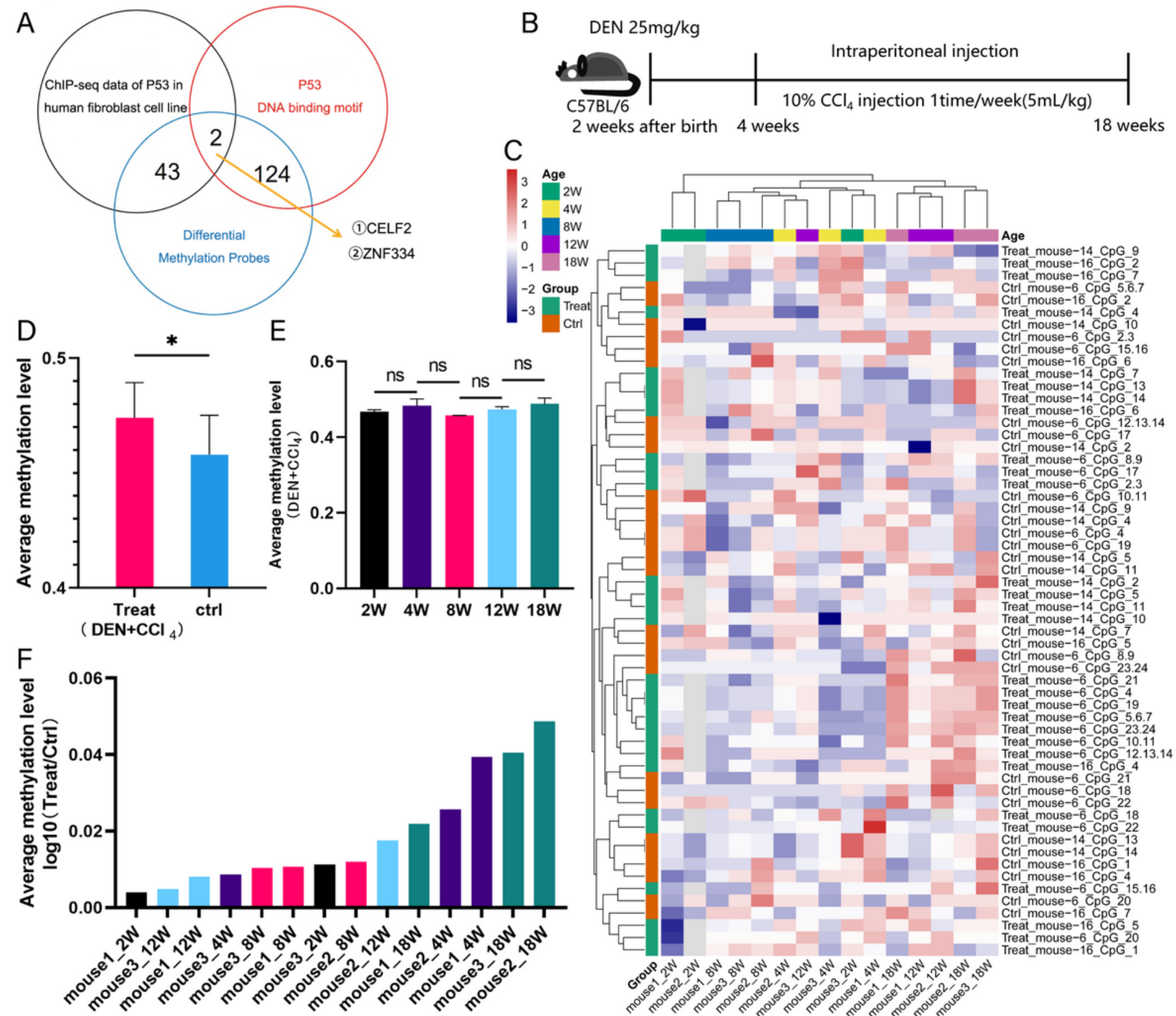

2 weeks after birth 4 weeks

18 weeks
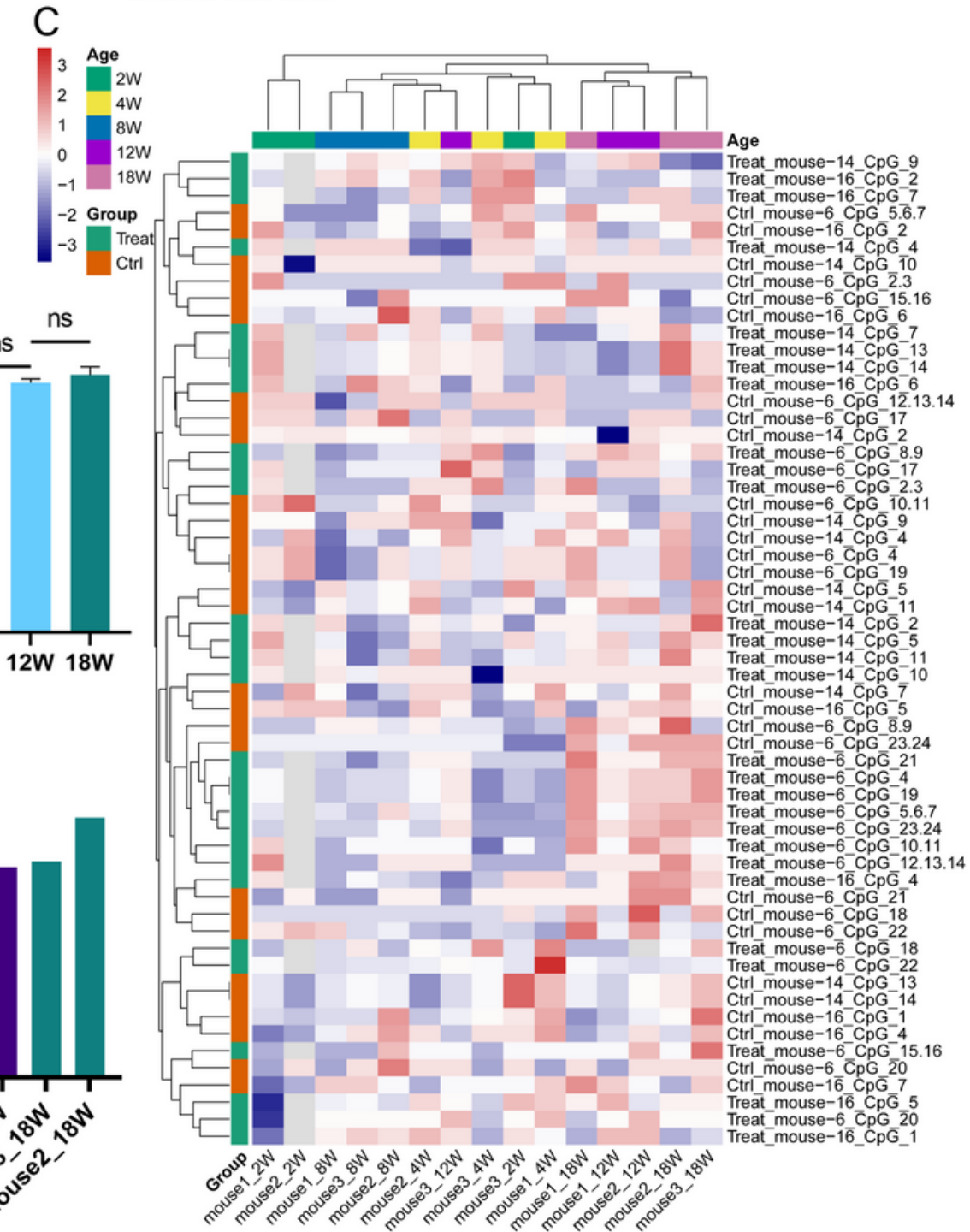

Figure 2

DEN-induced carcinogenesis mice model reveals hypermethylation of ZNF334 promoter. A. Venn diagram of the p53 target genes in three databases. B. Schematic diagram of DEN-induced carcinogenesis mice model. C. Heat map of methylation level of Zfp334 promoter in treatment and control group at indicated time. D. The average methylation degree of $\mathrm{Zfp} 334$ promoter in treatment and control group. E. The average methylation degree of $\mathrm{Zfp334}$ promoter in treatment group at indicated time. F. The ratio of methylation degree of Zfp334 promoter in treatment and control group (Treat/ctrl) at indicated time. 


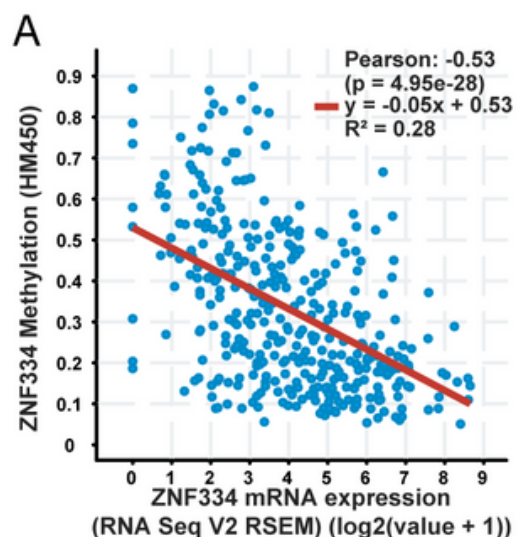

D

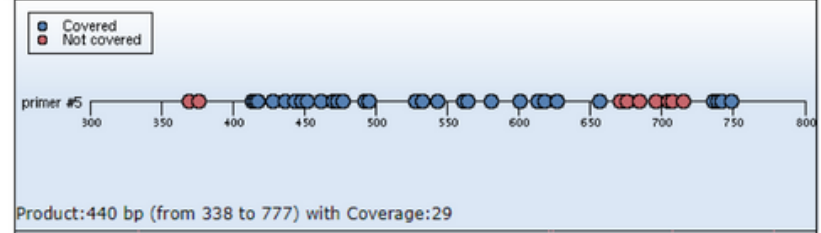

GGTCAGGAGCCCAATCCTGCCCTGGCTGCCTCGCCTCCCGACCACAGGAA ACCCTCCCTCCAAAGACAGGGAGAGCGCGCGTCCACCCTCCGCAGACCC GAGGCCCGCTCCGCCCGGCCCCACCGTTCCCTCCGACGCCCGAGTGCTC AGAGCTCGCCGCCAGCCCTTCCTATAGGTTGTGGGTGCACCCGCTTCGCC TCCAGTTCGCACCAGGTCCAAGTCTCGGCGGGACCAGGGATTCACGGGC TGCAGGACCCTCACCCGGAAGAGCCTGGCGAACCGGAAGGGCGTCCAG GTAAAAAACAGGAACAGCACATGCGCAGAAAGGCCCCCGGGCCGCCTTT CCCGGGGCTCTCCCGGGCCCCCCGCCGGCTTTCCGGACCACAGTCCCCAG AAGGCGTCGCGGCCACCGCCCTACCCTGAGGGATCTTTGAGGAGCT

$\mathrm{F}$

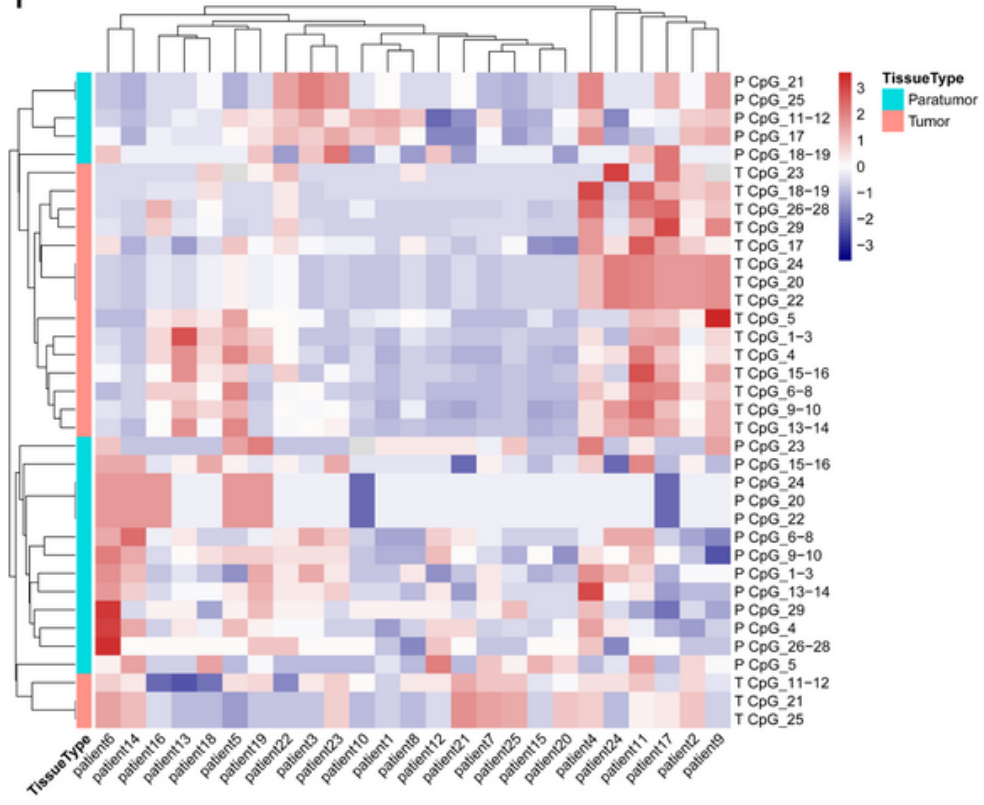

E

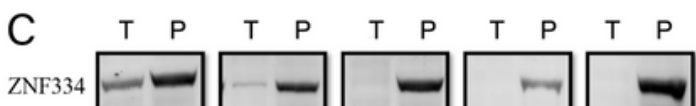

GAPDH
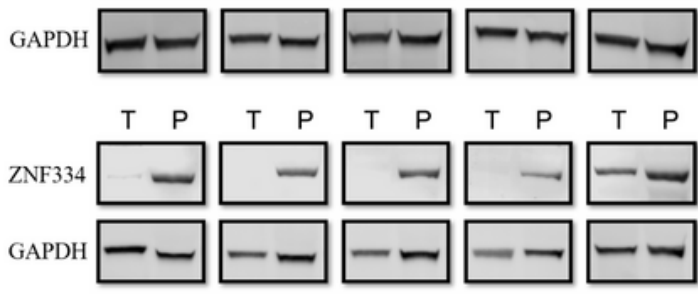
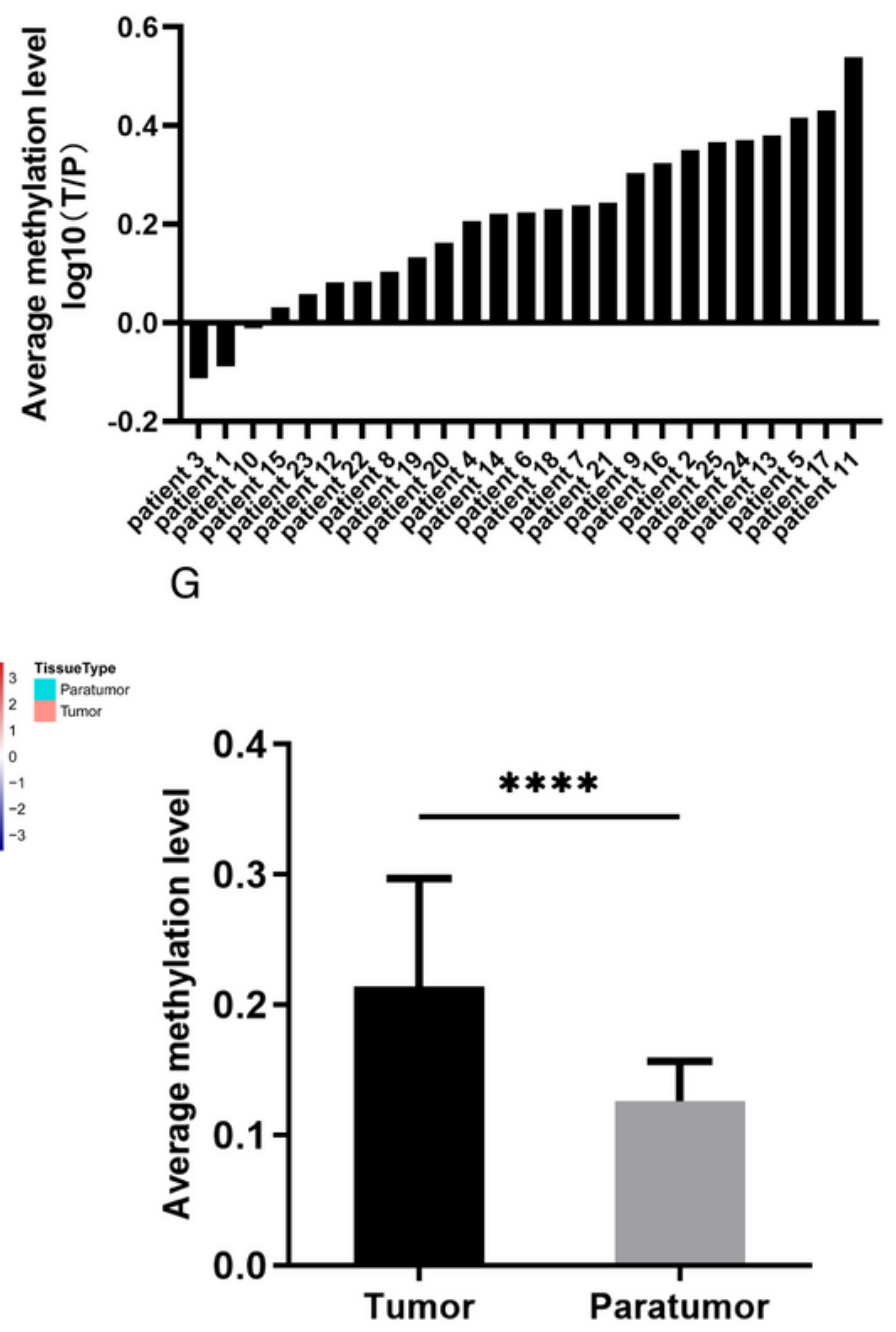

Figure 3

Promoter hypermethylation and expression of ZNF334 in HCC patients. A. A negative correlation between ZNF334 methylation level and its mRNA expression (Pearson:-0.53, R2=0.28) B. mRNA expression of ZNF334 in cancer and adjacent tissues of 213 liver cancer patients. C. Representative western-blot diagram of ZNF334 expression in cancer and adjacent tissues of 213 liver cancer patients. D. Methylation mass spectrum primers of ZNF334 promoter region and mass spectrometry detection product sequence 
(green indicates the EPIC chip probe cg07139762 site, which is in the ZNF334 promoter region; yellow indicates the MassARRAY methylation mass spectrometry detection sites, a total of 29). E. The ratio of methylation degree of ZNF334 promoter in cancer and adjacent tissues (T/P) of 25 patients with liver cancer. F. Heat map of methylation level of ZNF334 promoter region in cancer and adjacent tissues of 25 patients with liver cancer. G. The average methylation degree of ZNF334 promoter in cancer and adjacent tissues of 25 patients with liver cancer. ${ }^{* * *}, \mathrm{p}<0.0001$.

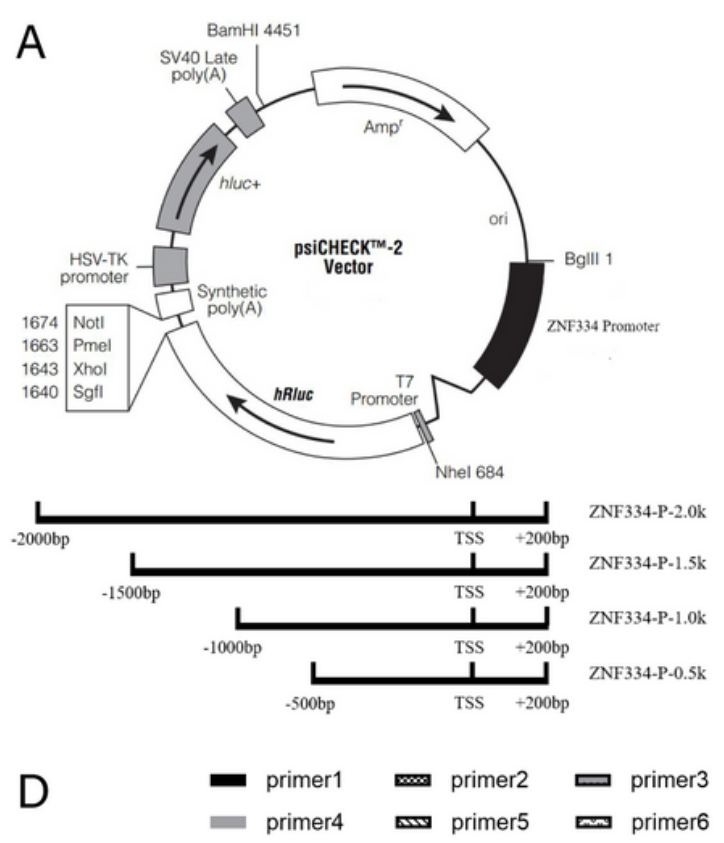

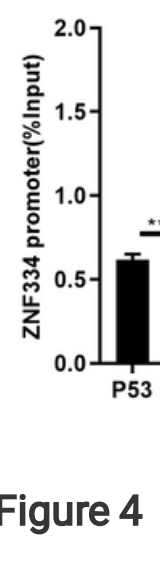

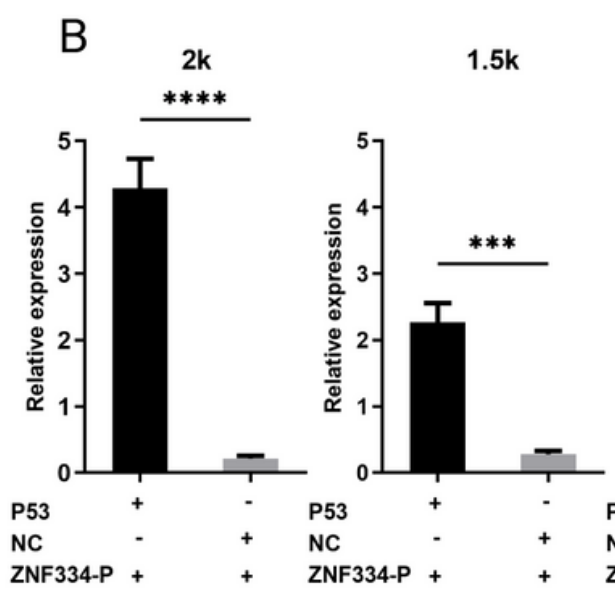

C Prediction of binding sites between P53 and the promoter of ZNF334

\begin{tabular}{cccccc}
\hline Name & Sequence & $\begin{array}{c}\text { Position } \\
(\text { 0-based })\end{array}$ & Strand & Score & P-value \\
\hline P53 & CATGTCT & 684 & + & 10.9 & 0.000575 \\
P53 & CATGCCT & 970 & + & 10.9 & 0.000575 \\
P53 & ATGTTTAGATATGTTT & 1134 & + & 16.14 & 0.00035 \\
P53 & TGACATGCC & 1199 & + & 12.4 & 0.000125 \\
P53 & TCGGATATCTCTGGGAATTG & 1555 & + & 4.01 & 0.000525 \\
P53 & GGGGGCCTTTCTGCGCATGT & 1952 & + & 4.87 & 0.000325 \\
\hline
\end{tabular}

E
$1 k$

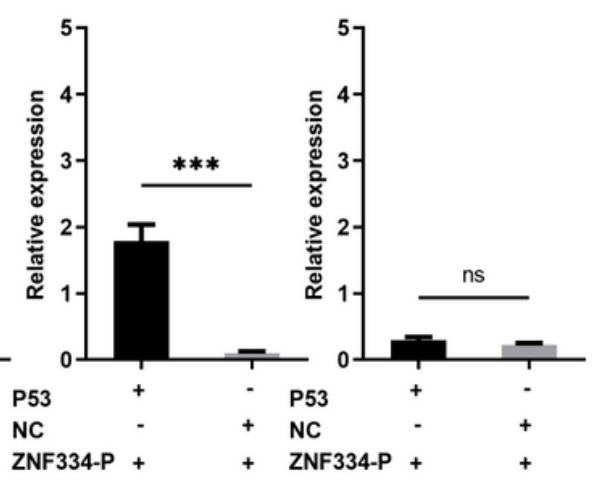

1952 0.000325

Transcription factor p53 can regulate the expression of TSG ZNF334. A. Diagram of the construction of dual luciferase plasmid (up) and diagram of truncation of the promoter region (down). B. Fluorescence intensity expressed by dual luciferin of plasmids with different lengths of ZNF334 promoter region. C. Prediction of binding sites between p53 and ZNF334 promoter region ( $p 53$ binding site sequence GGGGGCCTTTCTGCGCATGT contains the EPIC chip probe cg07139762). D. Conditions of p53 binding to different ZNF334 promoter sites. E. Electrophoresis diagram of PCR product by chromatin immunoprecipitation experiment. ***, $p<0.001 ; * \star \star \star, p<0.0001$. 


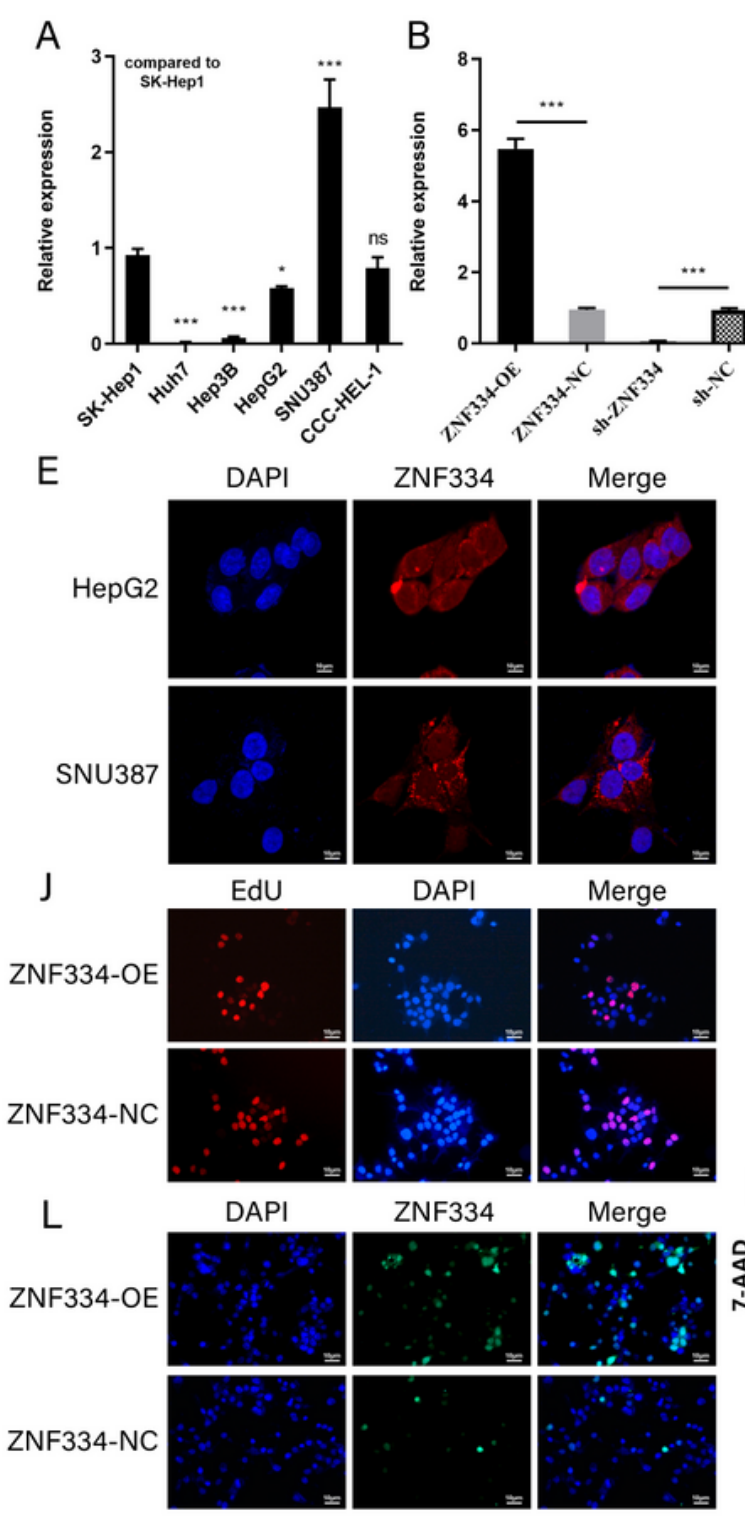

M ZNF334-OE ZNF334-NC

C

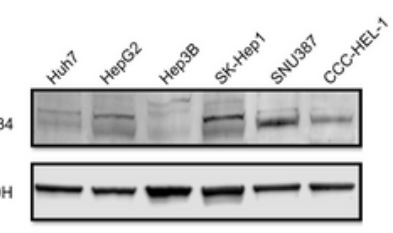

$\mathrm{F}$

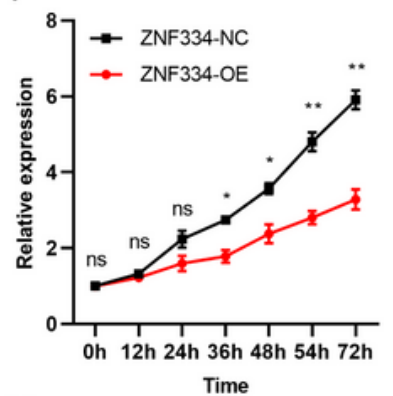

$\mathrm{H}$

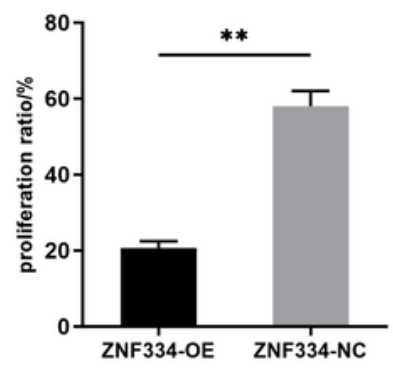

$\mathrm{K}$
D

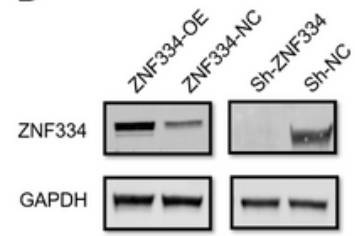

G

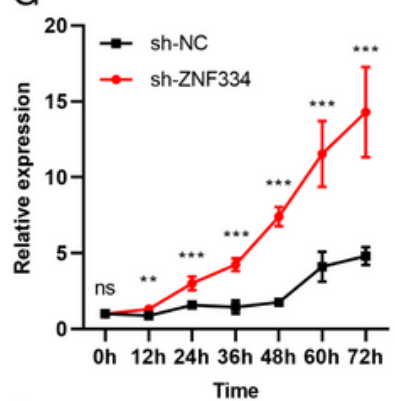

I

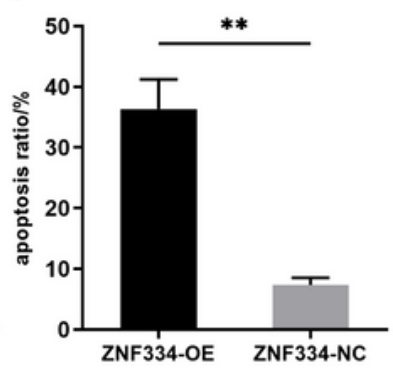

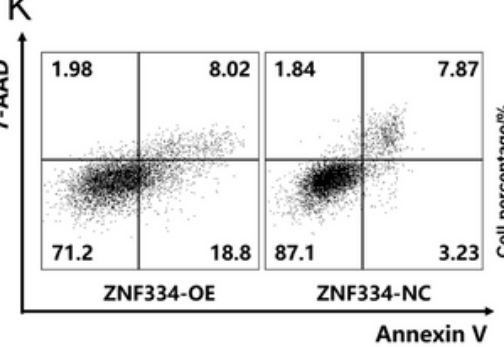

N
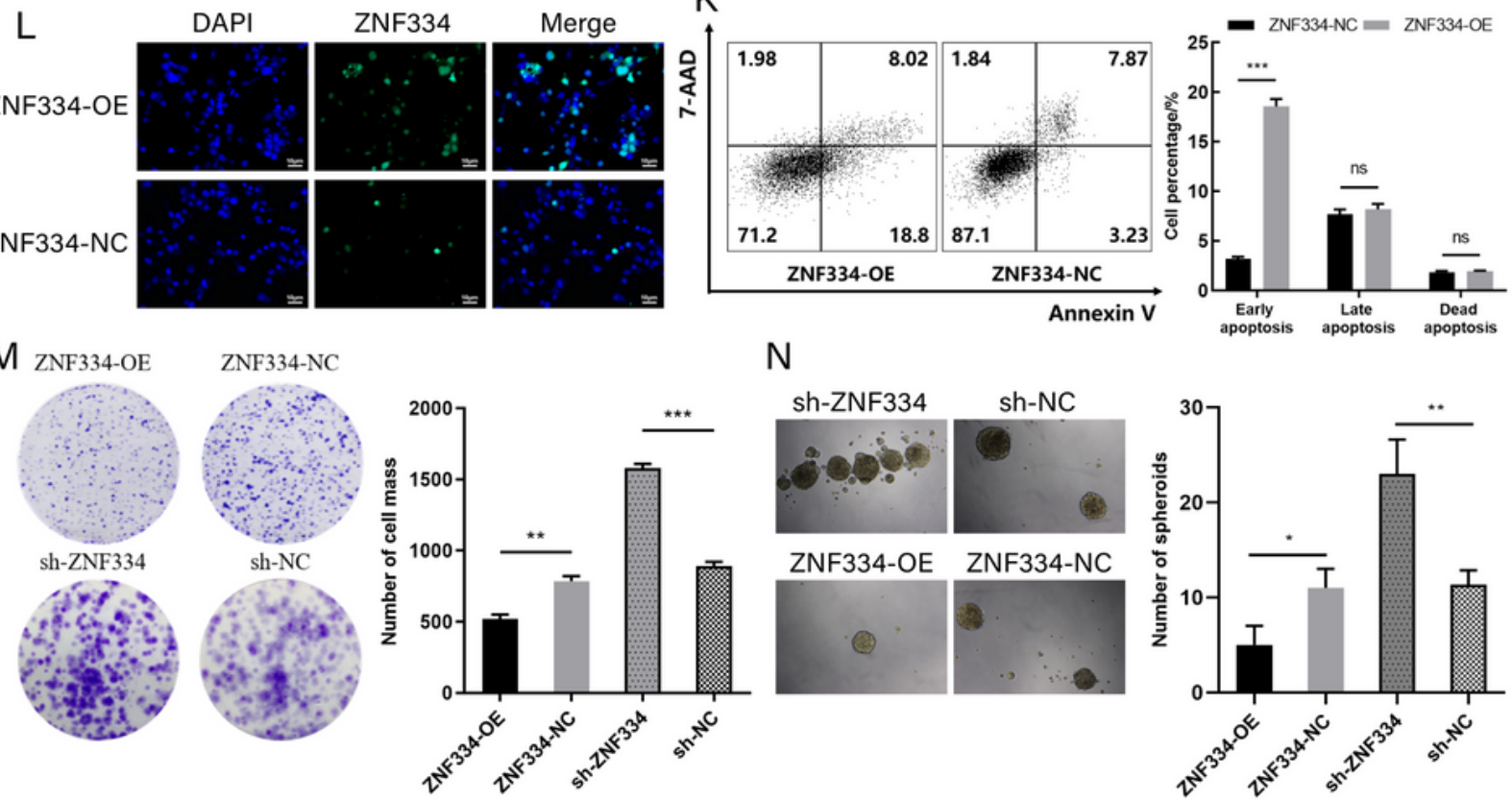

Figure 5

ZNF334 can promote cell apoptosis, inhibit proliferation and stemness of liver cancer cells in vitro. A. mRNA expression of ZNF334 in different cell lines. B. mRNA expression of ZNF334 in overexpression cell line (ZNF334-OE) and interfering cell line(sh-ZNF334). C. Protein expression of ZNF334 in different cell lines. D. Protein expression of ZNF334 in overexpression cell line (ZNF334-OE) and interfering cell line(shZNF334). E. Representative images of ZNF334 expression in HepG2, SNU387 (immunofluorescence 
staining, 200x, blue fluorescence represents nucleus, red fluorescence represents ZNF334). F. Cell proliferation curve of ZNF334-OE and ZNF334-NC by CCK8. G. Cell proliferation curve of sh-ZNF334 and sh-NC by CCK8. H. Proliferation ratio of ZNF334-OE and ZNF334-NC by EdU. I. Apoptosis ratio of ZNF334OE and ZNF334-NC by TUNEL. J. Representative images of proliferation of ZNF334-OE and ZNF334-NC by EdU (immunofluorescence staining, 200x, blue fluorescence represents nucleus, red fluorescence represents proliferating cells). K. Cell apoptosis of ZNF334-OE and ZNF334-NC by flow cytometry. L. Representative images of apoptosis of ZNF334-OE and ZNF334-NC by EdU (immunofluorescence staining, 200x, blue fluorescence represents nucleus, green fluorescence represents nucleus undergoing apoptosis). M. Representative images and statistical analysis of ZNF334-OE, ZNF334-NC, sh-ZNF334, and sh-NC by plate clone formation assay. N. Representative images and statistical analysis of ZNF334OE, ZNF334-NC, sh-ZNF334, and sh-NC by sphere formation assay. ns, not significant; *, $p<0.05$; **, $p<$ $0.01 ; * * *, p<0.001$.

A

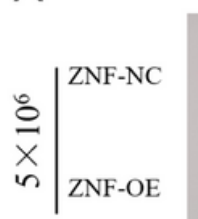

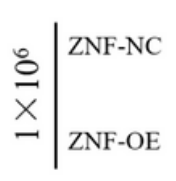

\begin{tabular}{l|l} 
ZNF-NC & \multirow{2}{*}{} \\
\hdashline & ZNF-OE
\end{tabular}

\begin{tabular}{l|l} 
ZNF-NC \\
$\bar{x}$ & ZNF-OE
\end{tabular}

D

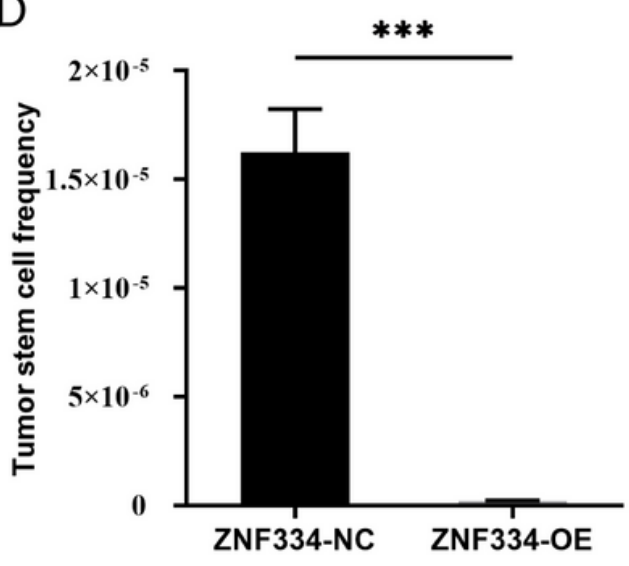

B

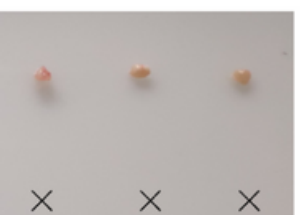

$\times$

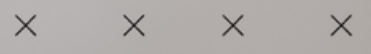

C

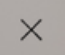

\begin{tabular}{|c|c|c|c|c|c|c|c|c|c|}
\hline \multirow{2}{*}{\multicolumn{2}{|c|}{$\begin{array}{l}\text { Frequency of colony } \\
\text { formation }\end{array}$}} & \multicolumn{4}{|c|}{$\begin{array}{c}\text { Number of cells seeded } \\
\text { each well }\end{array}$} & \multicolumn{3}{|c|}{$\begin{array}{l}\text { Confidence intervals for } \\
\text { stem cell frequency }\end{array}$} & \multirow{2}{*}{$P$ value } \\
\hline & & 100 & 200 & 400 & 800 & Estimate & Lower & Upper & \\
\hline \multirow{2}{*}{ Huh7 } & ZNF334-NC & $14 / 48$ & $18 / 24$ & $12 / 12$ & $6 / 6$ & $1 / 188$ & $1 / 254$ & $1 / 139$ & \multirow{2}{*}{$2.13 \mathrm{e}-04$} \\
\hline & ZNF334-OE & $4 / 48$ & $8 / 24$ & $11 / 12$ & $5 / 6$ & $1 / 467$ & $1 / 677$ & $1 / 322$ & \\
\hline \multirow{2}{*}{ SNU387 } & sh-ZNF334 & $27 / 48$ & $20 / 24$ & $12 / 12$ & $6 / 6$ & $1 / 114$ & $1 / 152$ & $1 / 85.3$ & \multirow{2}{*}{$1.99 \mathrm{e}-03$} \\
\hline & sh-NC & $13 / 48$ & $16 / 24$ & $11 / 12$ & $6 / 6$ & $1 / 223$ & $1 / 303$ & $1 / 163.4$ & \\
\hline
\end{tabular}

\begin{tabular}{|c|c|c|c|c|c|c|c|}
\hline \multirow{2}{*}{$\begin{array}{l}\text { Huh7 xenograft } \\
\text { tumor }\end{array}$} & \multirow{2}{*}{$\begin{array}{c}\text { Time } \\
\text { (months) }\end{array}$} & \multicolumn{4}{|c|}{ Cells injected } & \multirow{2}{*}{ Total } & \multirow{2}{*}{$P$ value } \\
\hline & & $5 \times 10^{6}$ & $1 \times 10^{6}$ & $5 \times 10^{5}$ & $1 \times 10^{5}$ & & \\
\hline ZNF334-OE & 2 & $6 / 6$ & $5 / 6$ & $3 / 6$ & $1 / 6$ & $\begin{array}{c}15 / 24 \\
(62.5 \%)\end{array}$ & \multirow[t]{2}{*}{$8.7 \mathrm{e}-05$} \\
\hline ZNF334-NC & 2 & $3 / 6$ & $2 / 6$ & $1 / 6$ & $0 / 6$ & $6 / 24(25 \%)$ & \\
\hline
\end{tabular}

$\mathrm{E}$

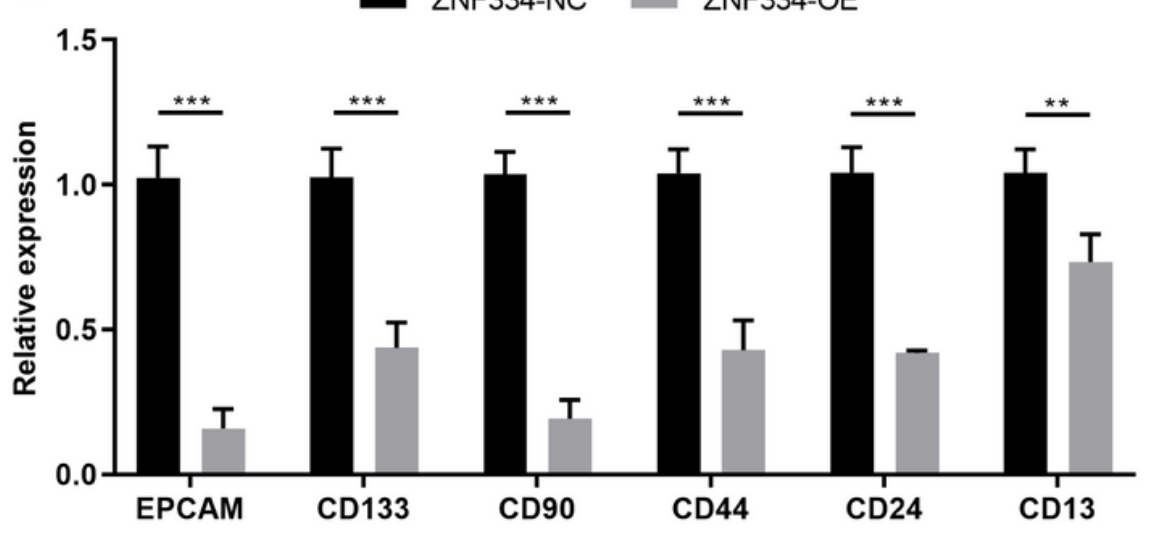

Figure 6 
ZNF334 can inhibit tumor formation in vivo. A. The number and size of subcutaneous tumors in nude mice with varying number of ZNF334-OE and ZNF334-NC. B. Extreme limiting dilution analysis of ZNF334-OE, ZNF334-NC, sh-ZNF334, and sh-NC. C. Extreme limiting dilution analysis in vivo. D. Proportion of tumor stem cells in subcutaneous tumor tissues of nude mice with ZNF334-OE and ZNF334-NC injected. E. Expression of different stem molecules of tumor stem cells in subcutaneous tumor tissues of nude mice with ZNF334-OE and ZNF334-NC injected. ***, $\mathrm{p}<0.001$.

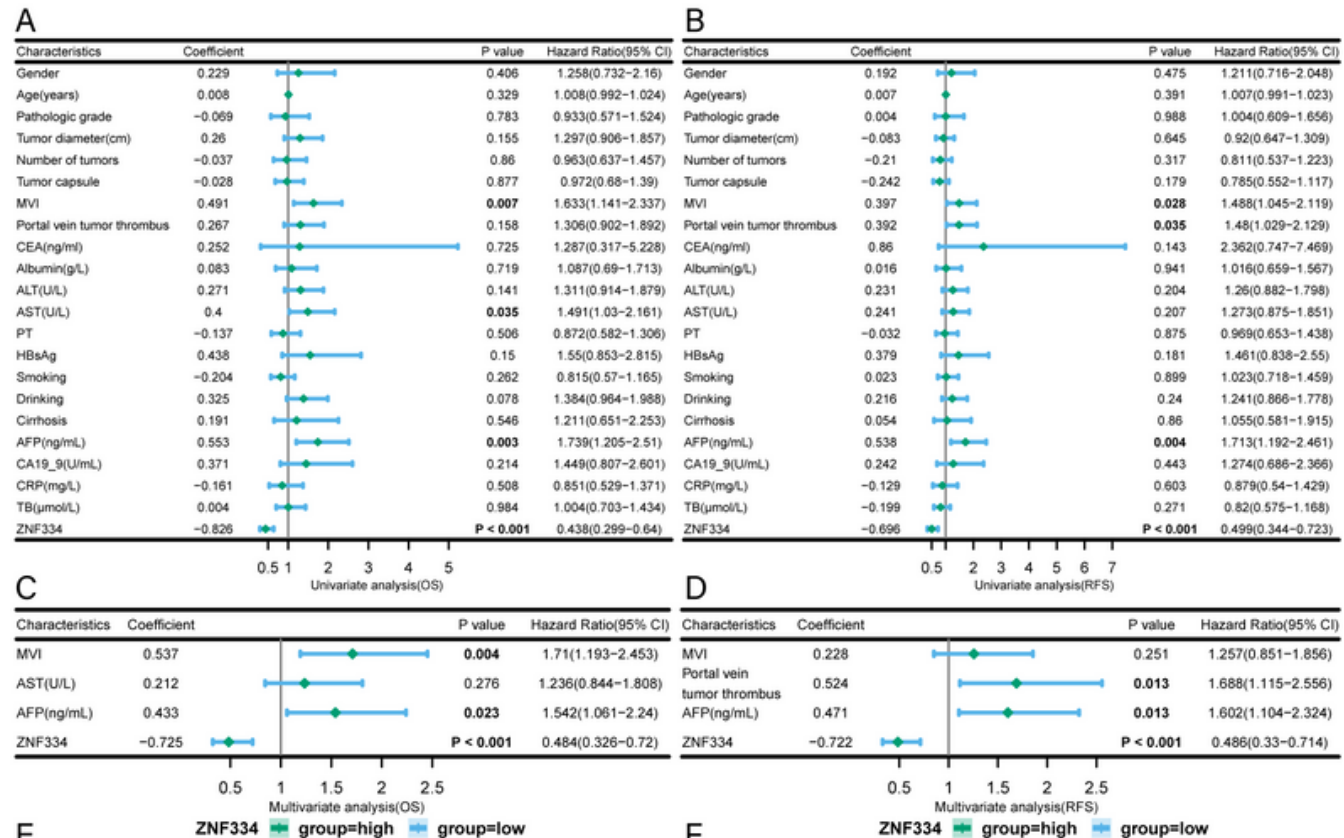

E

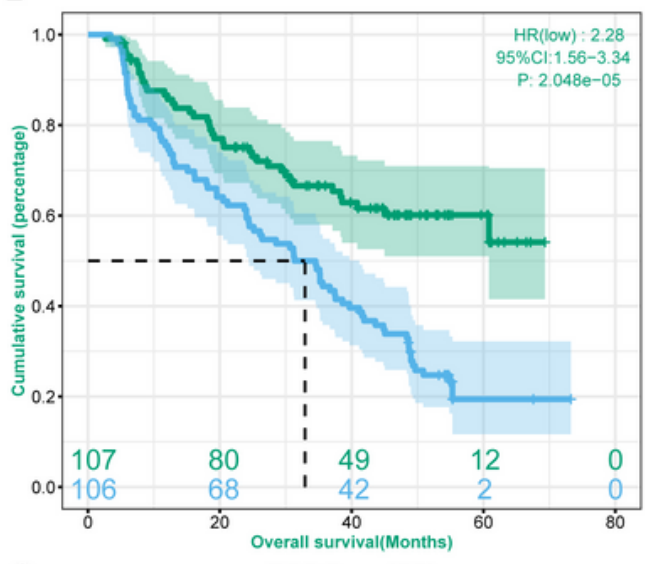

G

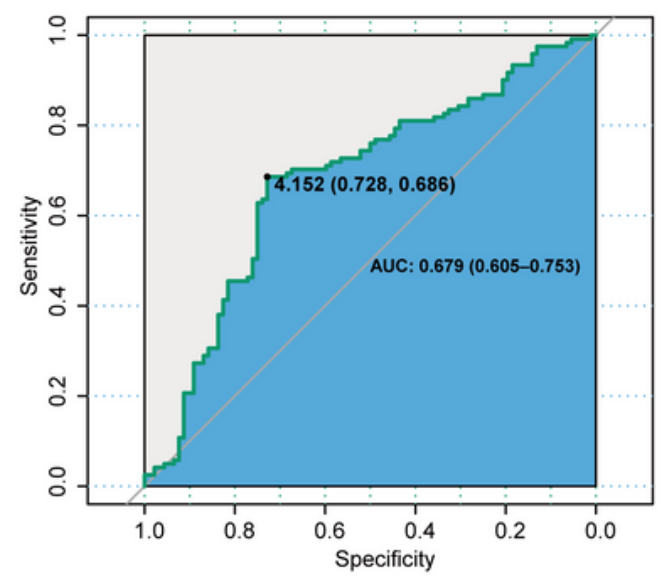

$\mathrm{F}$

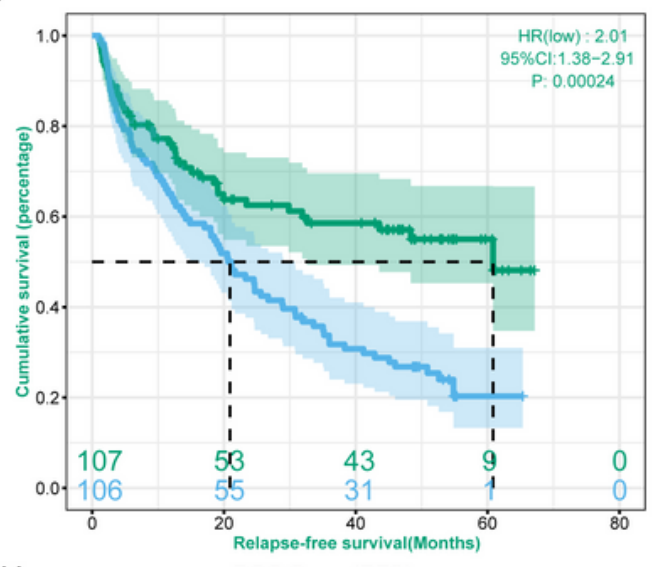

$\mathrm{H}$

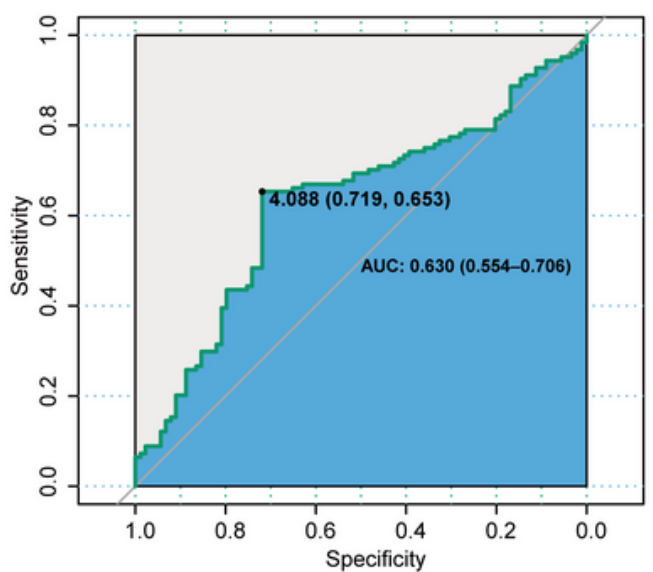

Figure 7 
The OS and DFS of patients with high expression of ZNF334 are longer. A. Univariate analysis of factors associated with overall survival of $213 \mathrm{HCC}$ patients. B. Univariate analysis of factors associated with relapse-free survival of $213 \mathrm{HCC}$ patients. C. Multivariate analysis of factors associated with overall survival of $213 \mathrm{HCC}$ patients. D. Multivariate analysis of factors associated with relapse-free survival of 213 HCC patients. E. Kaplan-Meier analysis showing the overall survival of HCC patients with diverse ZNF334 expression. F. Kaplan-Meier analysis showing the relapse-free survival of HCC patients with diverse ZNF334 expression. G. Receiver operating characteristics curve showing the ZNF334 cut-off value associated with overall survival. $\mathrm{H}$. Receiver operating characteristics curve showing the ZNF334 cut-off value associated with relapse-free survival.

\section{Supplementary Files}

This is a list of supplementary files associated with this preprint. Click to download.

- FigureS1.tif

- Figures2.tif

- SupplementaryTable1.docx 